\title{
The Dynamics of the Circumnuclear Disk and its environment in the Galactic Centre
}

\author{
B. Vollmer ${ }^{1}$ and W. J. Duschl ${ }^{2,1}$ \\ 1 Max-Planck-Institut für Radioastronomie, Auf dem Hügel 69, 53121 Bonn, Germany \\ 2 Institut für Theoretische Astrophysik der Universität Heidelberg, Tiergartenstraße 15, 69121 Heidelberg, \\ Germany
}

Received 27 July 2001 / Accepted 19 March 2002

\begin{abstract}
We address the question of the dynamics in the inner $50 \mathrm{pc}$ of the Galactic Centre. In a first step we investigate the cloud-cloud collision rate in the Circumnuclear Disk (CND) with the help of a three dimensional $N$-body code using gas particles that can have inelastic collisions. The CND might be a longer lived structure than previously assumed. The whole disk-like structure of the CND can thus survive for several million years. A realistic simulation of the CND shows the observed disk height structure. In a second step the environment of the CND is taken into account. Retrograde and prograde encounters of a cloud of several $10^{4} M_{\odot}$ falling onto an already existing nuclear disk using different energy loss rates per collision are simulated. The influence of the energy loss rate per collision on the evolution of the mass accretion and cloud collision rates is strongest for a prograde encounter. A composite data cube of two different snapshots of a prograde encounter together with the CND shows striking similarity with the observed Sgr A cloud complex. The current appearance of the Galactic Centre environment can thus be explained by at least two dynamically distinct features together with the CND. The current mass accretion rate within the CND ranges between $10^{-3}$ and $10^{-4} M_{\odot} \mathrm{yr}^{-1}$. It can rise up to several $10^{-2} M_{\odot} \mathrm{yr}^{-1}$ during massive accretion events.
\end{abstract}

Key words. galaxy: center - ISM: clouds - ISM: kinematics and dynamics

\section{Introduction}

The Galactic Centre region is an ideal laboratory for studying the fueling of a central black hole in great detail. Eckart \& Genzel (1996) have observed the proper motions of bright stars in the central few arcseconds around the non-thermal radio continuum source Sgr $\mathrm{A}^{*}$. They concluded that there is strong evidence for a $\sim 2.5 \times 10^{6} M_{\odot}$ central dark mass located within $\leq 0.015$ pc of Sgr A* with a mass density of at least $6.5 \times 10^{9} M_{\odot} \mathrm{pc}^{-3}$. This high density excludes the possibility that the central mass concentration is a central star cluster. Sgr A* is surrounded by a huge Hit region Sgr A West with a $\operatorname{size}^{1}$ of $2.1 \times 2.9 \mathrm{pc}$, which was first observed by Ekers et al. (1975). The ionized gas in this region forms a spiral pattern (see e.g. Lo \& Claussen 1983; Lacy et al. 1991) and is therefore called the Minispiral. The western side of this feature represents the inner edge of a large ring of molecular and atomic gas, the Circumnuclear Disk (CND) which extends from $\sim 2$ to $\sim 7$ pc from the centre. It was observed by several authors: Gatley et al. (1986) $\left(\mathrm{H}_{2}\right)$, Serabyn et al. (1986)

\footnotetext{
Send offprint requests to: B. Vollmer,

e-mail: bvollmer@mpifr-bonn.mpg.de

${ }^{1}$ We assume $8.5 \mathrm{kpc}$ for the distance to the Galactic Centre.
}

(CO, CS), Güsten et al. (1987) (HCN), DePoy et al. (1989) $\left(\mathrm{H}_{2}\right)$, Sutton et al. (1990) (CO), Jackson et al. (1993) (OI, HCN), Marr et al. (1993) (HCN), Coil \& Ho (1999, 2000) $\left(\mathrm{NH}_{3}\right)$, and Wright et al. (2001) (HCN). The main results of their investigations are:

- the CND has a hydrogen mass of a few $10^{4} M_{\odot}$,

- the disk is very clumpy with an estimated volume filling factor of $\Phi_{V} \sim 0.01$,

- the clumps have masses of $\sim 30 M_{\odot}$, sizes of $\sim 0.1 \mathrm{pc}$, and temperatures $\geq 100 \mathrm{~K}$.

It is now clear that the CND is interacting with the surrounding molecular clouds, but it is still a matter of debate where these connections are located (Zylka et al. 1990; Coil \& Ho 1999, 2000; Wright et al. 2001).

Krolik \& Begelman (1988) constructed a clumpy disk model for AGNs where cloud-cloud collisions are responsible for the energy and momentum transport and thus for the viscosity. Shlosman et al. (1990) discussed the possibility of fueling an AGN by the means of a cloudy disk. Sanders (1998) investigated the accretion process in the Galactic Centre on the basis of "sticky particle" calculations. He concluded that the gas features observed within 10 pc from the Galactic Centre can be understood in terms 
of tidal capture and disruption of gas clouds on low angular momentum orbits in a potential containing a point mass. The inner edge of the CND is explained by the formation of a "dispersion ring", an asymmetric elliptical torus precessing counter to the direction of rotation. This feature can be maintained for many orbital periods. These calculations follow the orbital motions of 4000 particles, whereas the number of observed clouds in the CND is $\sim 500$. Less clouds means less collisions and thus a lower viscosity (see e.g. Pringle 1981). This has influences on the way the dispersion ring forms.

In a previous article, we constructed a self-consistent model to describe the physical and kinematical state of the CND (Vollmer \& Duschl 2001a). The gas clouds were described as isothermal spheres embedded in an HiI region. The disk structure formed by the clouds was described by a quasi standard continuous accretion disk using adequately averaged parameters of the discrete cloud model. We succeeded in reproducing observed quantities as the cloud mass, the cloud radius, the electron density at the cloud edge, the electron density of the His region, the emission measure, the disk height, and the dispersion velocity. A major result was that the collisional time scale for one cloud is several Myr. The isolated CND might thus be much longer lived than previously assumed (Güsten et al. 1987).

In Vollmer \& Duschl (2001b) we pointed out a possibility to create the inner edge of the CND: as the clouds approach the Galactic Centre only those with densities high enough to resist tidal disruption can survive. At a certain distance from the Galactic Centre, the clouds with high enough densities to survive become too heavy and will fragment and/or collapse. This critical distance depends on the radiation field because it determines the radius of the clouds. Assuming realistic conditions for the Galactic Centre, we ended up with an inner edge at $\sim 2 \mathrm{pc}$.

The present article is devoted to the dynamics of the gas clouds in the CND. With the help of an improved numerical 3D collisional cloud model we investigate the collisional time scales of the CND, which is assumed not to interact with the surrounding molecular clouds. A more realistic way to model the Galactic Centre region is to include the molecular clouds, whose projected distances to the CND are small (see e.g. Mezger et al. 1989; Zylka et al. 1990). In the second part of this article we take this environment into account in our model and deduce more realistic collision rates for a gas clouds falling into the Galactic Centre with an already existing CND. A possible scenario for the star formation history in the Galactic Centre is presented.

\section{The model}

The numerical simulations must take into account that the CND consists of several hundred gas clouds. The present code treats each cloud as a point with a given mass and radius. The mass-radius relation for the clouds might be given by the virial theorem

$r_{\mathrm{cl}}=0.478 \times M_{\mathrm{cl}}\left[M_{\odot}\right] / T_{\mathrm{cl}}[\mathrm{K}] \mathrm{pc}$

where $r_{\mathrm{cl}}$ is the cloud radius, $M_{\mathrm{cl}}$ the cloud mass, and $T_{\mathrm{cl}}$ the cloud temperature. The temperature distribution with respect to the distance from the Galactic Centre is assumed to be

$T_{\mathrm{cl}}(R)=224 / \sqrt{R[\mathrm{pc}]} \mathrm{K}$

where $R$ is the distance of the cloud from the Galactic Centre. This distribution is consistent with the observed UV radiation field (Vollmer \& Duschl 2001a) and the deduced gas temperature (see e.g. Güsten et al. 1987; Sutton et al. 1990). On the other hand, Vollmer \& Duschl (2001a) have shown that the clouds in the CND have a constant radius $r_{\mathrm{cl}} \simeq 0.05 \mathrm{pc}$ if one takes the ionization front due to the external UV radiation field into account. In the present study we use both mass-radius relations.

We follow the orbits of these clouds in the three dimensional gravitational potential. The radial distribution of the total enclosed mass $M(R)$ is given by

$M(R)=M+M_{0} R^{\frac{5}{4}}$

where $M=3 \times 10^{6} M_{\odot}$ is the mass of the central black hole, and $M_{0}=1.6 \times 10^{6} M_{\odot} / \mathrm{pc}^{\frac{5}{4}}$ describes the mass distribution of the stellar content. This is close to the findings of Eckart \& Genzel (1996). When orbiting around the Galactic Centre, the clouds can have inelastic collisions. For the search of the next neighbour of a cloud a Barnes \& Hut (1986) treecode is used. During these collisions clouds can exchange mass and larger clouds can grow through coalescence. The result of a collision can be one (coalescence), two (mass exchange), or many fragments (fragmentation). In our model, we limit the fragmentation case to a maximum of 3 fragments. Let the radius of the first cloud be $r_{1}$, that of the second cloud $r_{2}$. Let the impact parameter be $b$, the velocity of the fragment $v_{\mathrm{f}}$, and the escape velocity $v_{\text {esc }}$. We follow the prescriptions of Wiegel (1994):

$$
\begin{aligned}
- & \text { for } r_{1}-r_{2}<b<r_{1}+r_{2} \text { : } \\
& \text { fragmentation } \\
- & \text { for } b \leq r_{1}-r_{2} \text { and } v_{\mathrm{esc}}>v_{\mathrm{f}} \text { : } \\
& \text { mass exchange } \\
- & \text { for } b \leq r_{1}-r_{2} \text { and } v_{\mathrm{esc}} \leq v_{\mathrm{f}} \text { : } \\
& \text { coalescence. }
\end{aligned}
$$

In order to avoid the production of a large number of too small clumps $\left(M<10 M_{\odot}\right)$, we treat a fragmentary collision, which would give rise to a cloud with $M<10 M_{\odot}$, as a mass-exchange collision in adding the mass of the potential third cloud to that of the lightest colliding clouds. This procedure ensures that the arising cloud mass spectrum has a maximum between 10 and $15 \mathrm{M}_{\odot}$.

The integration of the ordinary differential equation is done with the Burlisch-Stoer method (Stoer \& Burlisch 1980) using a Richardson extrapolation and Stoermer's 
rule. This method advances a vector of dependent variables $y(x)$ from a point $x$ to a point $x+H$ by a sequence of $n$ substeps. Thus, the initial timestep $H$ is divided subsequently into $n=2,3,4$, etc. substeps. At the end the solution of $y(x+H)$ is extrapolated and an error can be estimated. The size of the timestep is adaptive and linked to the estimated error of the extrapolation. This error is normalized by the values of the distance covered during the last timestep and the velocity of each particle. The error level for acceptance of the extrapolated solution is a free parameter and has to be adapted to the physical problem treated. For a relative error level $\Delta r_{i} / r_{\text {norm }}=\Delta v_{i} / v_{\text {norm }}<0.1$, where $i=1$, $2,3, r_{\text {norm }}=10^{-2} \mathrm{pc}$ and $v_{\text {norm }}=5 \times 10^{-2} \mathrm{~km} \mathrm{~s}^{-1}$ the Courant criterion is fulfilled for each timestep.

The collisions are evaluated at each timestep $h=H / n$ and only those which appear for all sequences $n$ are taken into account. Therefore, the relative error level $\epsilon$ is crucial for the value of the obtained collision rates. We adopt the strategy to choose the error level in a way to match the theoretical collision rates.

\section{The normalization of the collision rate}

With the fixed precision of the calculations it is possible to compare the model collision time for one cloud with the theoretical one:

$t_{\mathrm{coll}} \simeq\left(n_{\mathrm{cl}} \sigma_{\mathrm{cl}} v_{\mathrm{cl}}\right)^{-1}$

where $n_{\mathrm{cl}}$ is the local cloud density, $\sigma_{\mathrm{cl}}=\pi r_{\mathrm{cl}}^{2}$ the cross section of the cloud, and $v_{\mathrm{cl}}$ the cloud velocity. The comparison of the mean collision time for one cloud are made with the simplest spatial configuration, namely a spherical volume. The clouds are initially distributed uniformly in a sphere with a radius of 4 pc. The initial velocities are uniformly distributed between $20 \mathrm{~km} \mathrm{~s}^{-1}$ and $40 \mathrm{~km} \mathrm{~s}^{-1}$. The gravitational potential is due to the following mass distribution: $M(R)=M_{0} R^{\frac{5}{4}}$ with $M_{0}$ from Eq. (3). In order to check our numerics, we made one set of simulations where the potential collisions are counted, but not executed. In this case there is only negligible angular momentum transfer due to gravitational interactions between the clouds and the spatial distribution of the clouds stays approximately constant during the simulation.

The influence of the mass-radius relation on the collision rate is tested with two different relations:

- RUN1: $r_{\mathrm{cl}} \propto M_{\mathrm{cl}} \sqrt{R}$. In this case the cloud temperature adjusts to the temperature given in Eq. (2). Since there is cloud heating due to cloud-cloud collisions, it is assumed that the dynamical time scale is much larger than the cooling time scale due to radiation.

- RUN2: $r_{\mathrm{cl}}=$ const. In this case an external UV radiation field is assumed that creates an ionization front on the cloud surface which is directed to the Galactic Centre. The location of this ionization front does not depend on the clouds' distance to the Galactic Centre (Vollmer \& Duschl 2001a), i.e. the cloud radius is constant.
The ratio of the model mean collision time of one cloud and the theoretical one for RUN1 and RUN2 without executed collisions is shown in Fig. 1. The theoretical mean collision time is given by

$t_{\mathrm{coll}}^{\text {theo }} \simeq\left\langle n_{\mathrm{cl}} \sigma_{\mathrm{cl}} v_{\mathrm{cl}}\right\rangle^{-1} \simeq\left(\left\langle n_{\mathrm{cl}}\right\rangle\left\langle\sigma_{\mathrm{cl}}\right\rangle\left\langle v_{\mathrm{cl}}\right\rangle\right)^{-1}$.

The ratio for both simulations stays constant over 100 Myr. It is $\sim 1$ for RUN1 and $\sim 0.75$ for RUN2. Thus, both simulations reproduce the theoretical collision time scale correctly within $25 \%$. The difference between RUN1 and RUN2 lies in the density gradient of the cloud distribution. Clouds at the outskirts of the sphere are larger for RUN1. Therefore, the average theoretical collision time scale is smaller, and the ratio between the model and the theoretical collision time scales is larger. Since these clouds are located in a region with a low particle density and do not contribute much to the global model collision time scale, the ratio between the model and theoretical collision time scale is larger.

We then calculated another set of simulations with proceeding cloud-cloud collisions. As the system evolves, the angular momentum transport through these collisions, i.e. the viscosity, makes the particle density increase in the centre. The initial and the final stage of such a simulation is shown in Fig. 2. The ratio between the model and theoretical mean collision time for RUN1 and RUN2 is shown in Fig. 3. The theoretical mean collision time is given by Eq. (5).

- RUN1: during 6 Myr the ratio between the model and theoretical collision time scales is $\sim 1$. As the spatial cloud distribution changes to higher central densities, the number of collisions in the system is overestimated by Eq. (5), i.e. the collisional time scale is underestimated. This makes the ratio between the model and the theoretical collision time scale increase. The critical cloud density for this increase is $n_{\mathrm{cl}} \sim 100$ clouds $/ \mathrm{pc}^{3}$.

- RUN2: we observe here the same trend as for RUN1. When the cloud distribution contracts, the number of collisions is theoretically underestimated. This effect also starts at a critical cloud density of $n_{\mathrm{cl}} \sim$ 100 clouds $/ \mathrm{pc}^{3}$.

We thus conclude that the mean collision time for one cloud, which is resulting from the model, reflects the real value or might underestimate it by $\sim 25 \%$ if the cloud distribution is not heavily concentrated.

\section{CND simulations as an isolated structure}

We have distributed 500 particles within a ring volume of constant height $H=1 \mathrm{pc}$ and an inner radius of $R_{\text {in }}=2 \mathrm{pc}$ and an outer radius $R_{\text {out }}=7 \mathrm{pc}$. The initial velocities were Keplerian using a gravitational potential made by the mass distribution described in Eq. (3). A turbulent velocity of $20 \%$ of the Keplerian value was added in an arbitrary direction. The system evolved then freely in the gravitational potential of a point mass (the central 

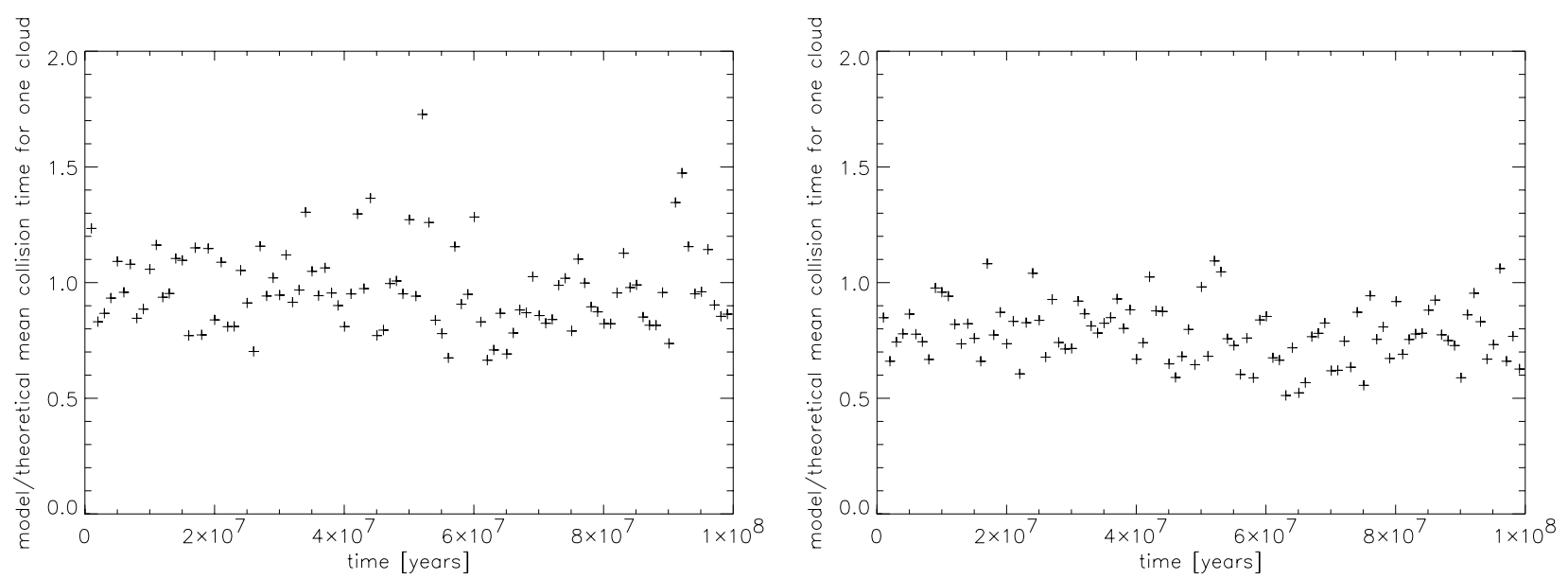

Fig. 1. The ratio between the model and theoretical mean collision time without executed collisions. Left panel: RUN1. Right panel: RUN2.
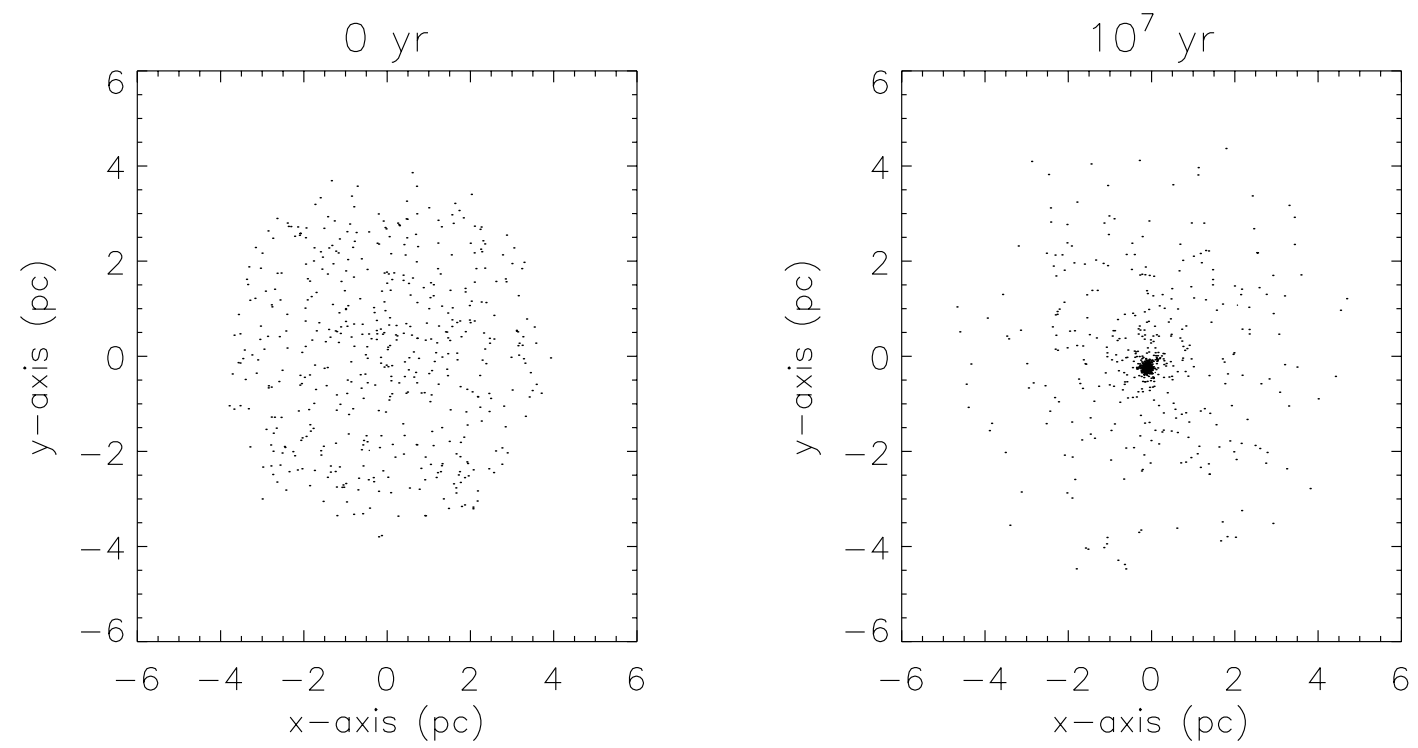

Fig. 2. Right: initial state of a simulation with collisions. Left: final state of the simulation after $10^{7} \mathrm{yr}$.
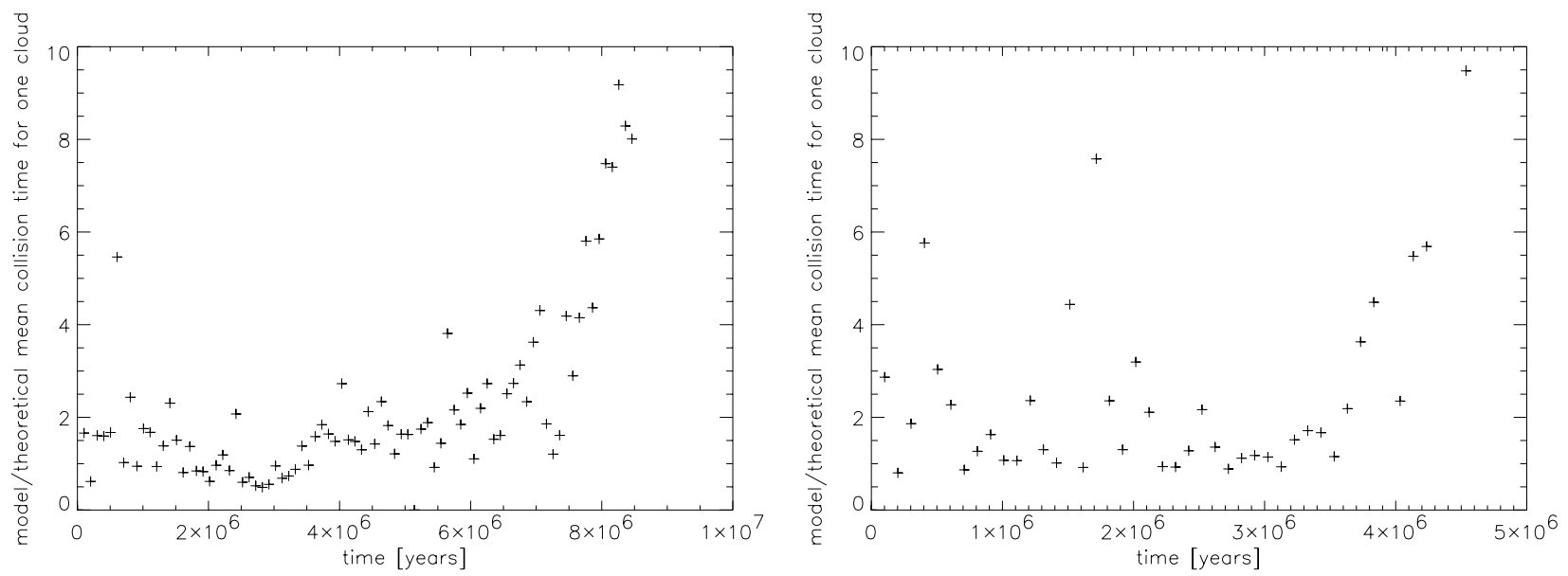

Fig. 3. The ratio of the model mean collision time to the theoretical one with proceeding collisions. Left panel: RUN1. Right panel: RUN2.

black hole) and an extended mass distribution (the central star cluster). Vollmer \& Duschl (2001b) have shown that the clouds, which approach the Galactic Centre, become tidally disrupted if they are not dense enough to 

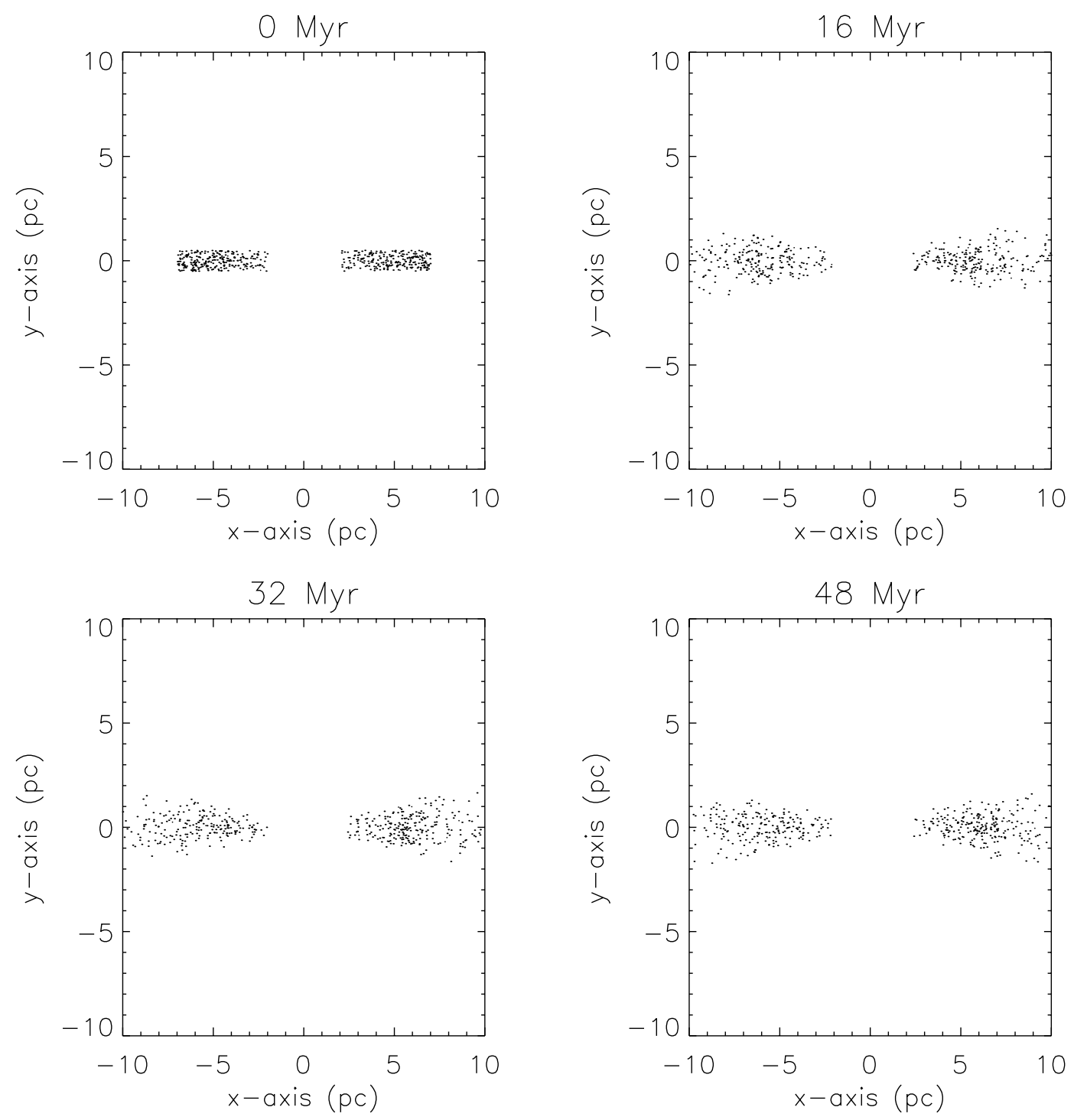

Fig. 4. The evolution of the cloudy disk model without collisions. The elapsed time is shown at the top of each frame. The $x$-axis shows the distance of the clouds to the centre, the $y$-axis shows the vertical distance with respect to the disk plane.

resist. Those clouds with a high enough central density to be stable at a radius of $R_{\text {crit }} \sim 2$ pc will collapse and/or fragment. In any case, no cloud can survive at radii closer than $R_{\text {crit }}$. In our model we take this effect into account by removing clouds with $R<R_{\text {crit }}$.

As in Sect. 3 we have made first a simulation counting the collisions without executing them. The cloud radius is $r_{\mathrm{cl}}=$ const. $=0.05$ pc. Figure 4 shows different states of evolution of such a disk-like structure. The timesteps are $\Delta t=16$ Myr. The first plot shows the ring-like initial condition. After several Myr the disk reaches a state of equilibrium. The disk thickness at a distance of $5 \mathrm{pc}$ is about 2 pc. The collisional time scale for one cloud is shown in Fig. 5. It ranges between 2 and $3 \mathrm{Myr}$.

In the next step we have made two simulations with collisions using the two different mass-radius relations RUN1 and RUN2. Since the evolution of the spatial cloud distribution is quasi identical for RUN1 and RUN2, we show only that of RUN2 in Fig. 6.

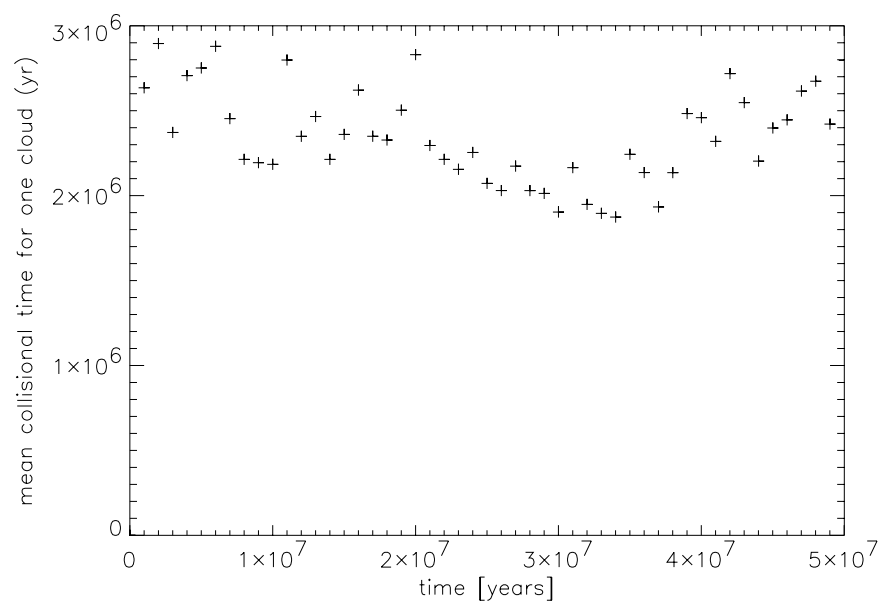

Fig. 5. The evolution of the collision time scale for one cloud for the simulation shown in Fig. 4. 

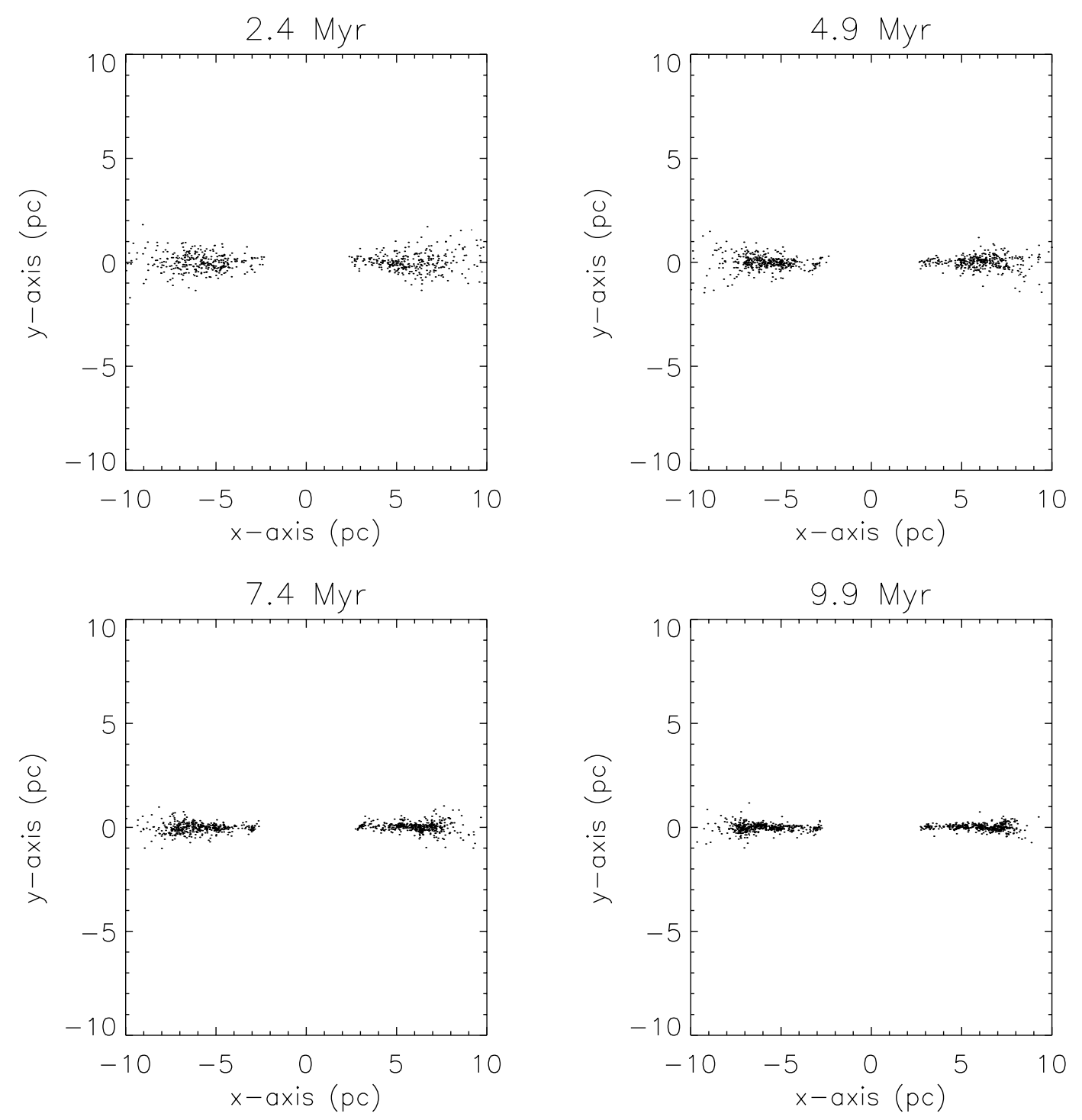

Fig. 6. The evolution of the cloudy disk model with collisions. The elapsed time is shown at the top of each frame. The $x$-axis shows the distance of the clouds to the centre, the $y$-axis shows the vertical distance with respect to the disk plane.

The observed thickness of the CND, i.e. $0.5 \mathrm{pc}$ at a distance of $2 \mathrm{pc}$ and $1.5 \mathrm{pc}$ at a distance of $5 \mathrm{pc}$ from the Galactic Centre (Güsten et al. 1987) lies between those of the model with and without collisions. The initial mass distribution $18 M_{\odot}<M_{\mathrm{cl}}<38 M_{\odot}$ (Fig. 7 dotted line) smears out due to the inelastic collisions. The mass distribution after $10 \mathrm{Myr}$ is plotted as a solid line in Fig. 7 . The maximum has shifted to smaller cloud masses and a high mass tail has been built. A part of these high mass clouds should have collapsed, but this mechanism is not included in our code.

The resulting evolution of the model mean collision time can be seen for the mass-radius relation RUN1 and RUN2 in Fig. 8. We observe an increasing mean collision time for the mass-radius relation RUN1 (Fig. 8 left panel). This is, because the cloud distribution is shifted to smaller masses. Thus the clouds are smaller and the collision time scale, which is proportional to the square of the cloud radius, increases. For the case of a constant radius independent of the cloud mass (RUN2; Fig. 8 right panel) the collision rate increases, because the number of clouds and thus the cloud density increases.

Since the cloud density is always smaller than $n_{\text {crit }}$ the results for the mean collision time scales is robust. The derived mean collision time scale for one cloud is of the order of 1-2 Myr.

In Vollmer \& Duschl (2001a) we gave a mean collision time scale of $\sim 10$ Myr. The factor 5 between both approaches can be understood in the light of the analytical model for turbulent, clumpy accretion disks (Vollmer \& Beckert 2002). In this model, the viscosity is given as $\nu=R e^{-1} v_{\text {turb }} l_{\text {driv }}$, where $v_{\text {turb }}$ is the turbulent velocity dispersion and $l_{\text {driv }}$ is the driving wavelength for the turbulence. From the analytical model it follows that $l_{\text {driv }}=H$, where $H$ is the disk height. Thus one obtains $\nu=R e^{-1} v_{\text {turb }} H$. This viscosity prescription is also used 


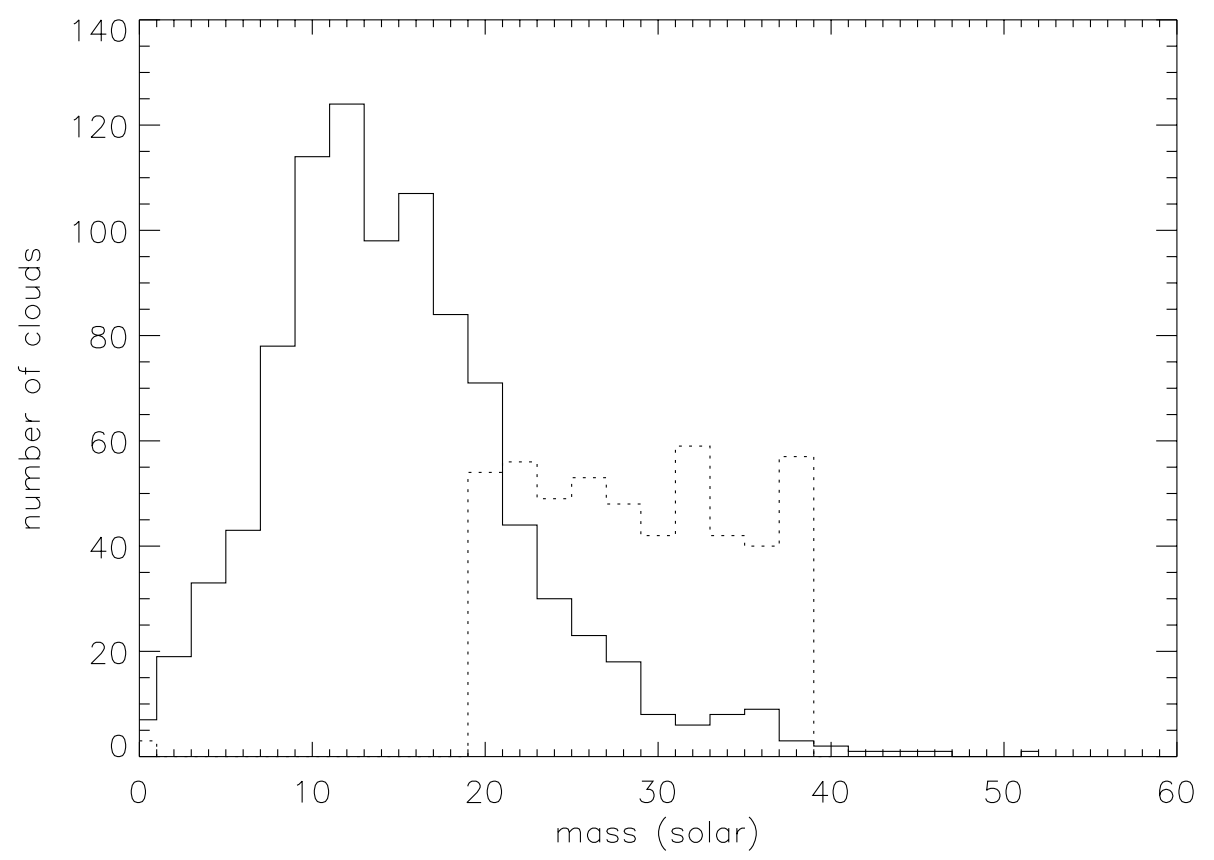

Fig. 7. The mass spectrum of the clouds. Dotted line: initial mass spectrum. Solid line: mass spectrum after 10 Myr.
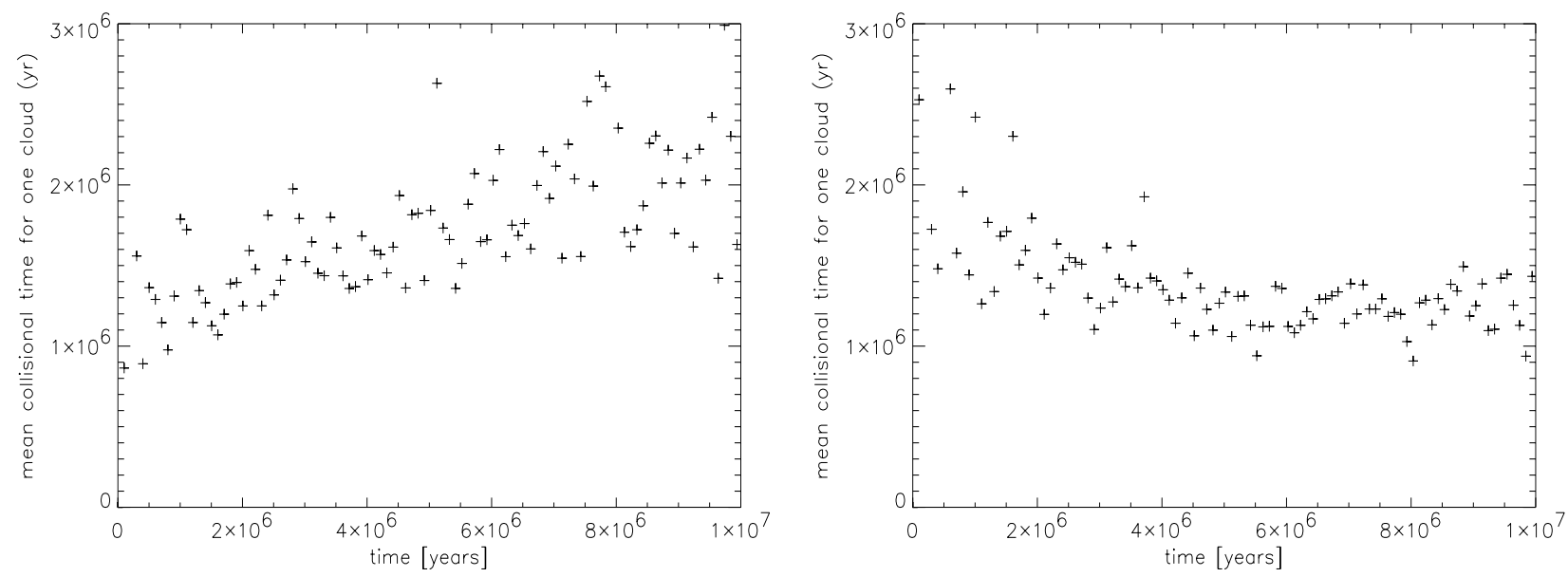

Fig. 8. Model mean collision times for the mass-radius relation Left panel: RUN1. Right panel: RUN2.

in Vollmer \& Duschl (2001a). The relation between the gas surface density $\Sigma$ and the mass accretion rate $\dot{M}$ of the disk is (see, e.g., Pringle 1981)

$3 \pi \nu \Sigma=\dot{M}$

The collision time scale for one cloud is then $t_{\text {coll }}=$ $R e \Omega^{-1}$. In Vollmer \& Duschl (2001a) we used $R e=1000$, whereas a Reynolds number of $R e=20$ has to be used in the framework of Vollmer \& Beckert (2002), which is consistent with our dynamical results (Fig. 8). Vollmer \& Duschl (2001a) assumed thus a relatively large Reynolds number. Consequently, they obtained a relatively large gas surface density (Eq. (6)), i.e. a relatively large disk mass $\left(M_{\text {gas }} \sim 1.5 \times 10^{5} M_{\odot}\right)$, about a factor of 10 larger than that what we have used here.

\section{Environmental effects}

In the previous sections we have treated the CND as an isolated structure. In reality this is not the case. Observations of molecular transitions (e.g. CO: Sutton et al. 1990; CO, CS: Serabyn et al. 1986; HCN: Coil \& Ho 1999, 2000; Wright et al. 2001) and mm observations (e.g. Mezger et al. 1989; Dent et al. 1993) have shown that there are several molecular cloud complexes in the vicinity of the Galactic Centre. Sgr A East Core, a compact giant molecular cloud with a gas mass of several $10^{5} M_{\odot}$ and the giant molecular clouds M-0.13-0.08 and M-0.02-0.07 form the Sgr A cloud complex. We will compare our simulations to the observational results of Zylka et al. (1990), because their distinction of different cloud complexes is made on kinematical grounds. They obtained the following results: M-0.13-0.08 has radial velocities in the range 
of $\sim 5-25 \mathrm{~km} \mathrm{~s}^{-1}$, a total mass of $\sim 3 \times 10^{5} M_{\odot}$ and lies in front of the Galactic Centre. M-0.02-0.07 consists of two different features. (i) Sgr East Core with a mass of $\sim 2 \times 10^{5} M_{\odot}$ and (ii) a curved streamer of total mass $\sim 10^{5} M_{\odot}$ and velocities between 25 and $65 \mathrm{~km} \mathrm{~s}^{-1}$, which lies also in front of the Galactic Centre. Thus, there is neutral gas with a mass of several $10^{5} M_{\odot}$, which is presumably located within the inner 50 pc around the Galactic Centre (see, however, von Linden et al. 1993). In addition, there are hints that they are kinematically connected to the CND.

\subsection{Initial conditions}

We let evolve a model of the CND as described above with 500 particles and a total mass of $\sim 1.5 \times 10^{4} M_{\odot}$ during $10 \mathrm{Myr}$. We then added a spherical mass distribution of 1000 particles, a total mass of $3 \times 10^{4} M_{\odot}$, and a size of $10 \mathrm{pc}$ at a distance of $\sim 30 \mathrm{pc}$. The initial values of the velocity of these clouds were $30 \%$ of the Keplerian value with an additional velocity dispersion of $20 \%$ of this value. Due to the sub-Keplerian velocity, the cloud falls into the Galactic Centre colliding with the CND. This initial configuration can be seen in Fig. 9. We have made two different sets of simulations:

(i) a prograde encounter, i.e. the orbital angular momentum of the cloud is close to that of the disk;

(ii) a retrograde encounter, i.e. the orbital angular momentum of the cloud is opposite to that of the disk. Technically, this is realized in mirroring the $z$-axis for the disk clouds: $z^{i}=-z^{i}, v_{z}^{i}=-v_{z}^{i}$, where $i$ is the number of the cloud.

Furthermore, we varied the fraction of kinetic energy that is radiated away during a cloud-cloud collision. This has important influences on the collision and mass accretion rate of the system.

\subsection{The model evolution}

\subsubsection{Retrograde encounter}

The evolution of a system with no loss of kinetic energy per collision can be seen in Fig. 10. The timestep is $\Delta t=1.2 \mathrm{Myr}$. The cloud hits the CND for the first time at $t \simeq 0.3 \mathrm{Myr}$. The second collision takes place at $t \simeq 0.8 \mathrm{Myr}$. The period of the orbit is $t_{\text {orb }} \sim 0.7 \mathrm{Myr}$. During its orbiting the cloud is stretched due to the tidal shear. The streamer becomes a filament of $\sim 40 \mathrm{pc}$ within $1 \mathrm{Myr}$. As the cloud collides for the first time with the CND, the latter is heavily damaged and shows an azimuthally asymmetric cloud distribution. Meanwhile, a counter rotating streamer is building up at the outer part of the CND. After $\sim 3 \mathrm{Myr}$ the CND becomes less and less prominent. After $\sim 2$ Myr the streamer crosses itself for the first time and has a "brezel" form at $t \sim 3 \mathrm{Myr}$. Later on, it begins to form a triangular feature. At the end of the simulation ( $t \sim 10 \mathrm{Myr}$ ), we observe this triangular feature containing a ring-like rotating structure extending from $\sim 5$ to $\sim 10 \mathrm{pc}$ and a counter rotating core (the former CND). The simulation with $10 \%$ energy loss per collision shows a more pronounced ring-like structure and a less prominent rotating core.

This evolution can be also described by the number of collisions and by the mass accretion rate. We define this mass accretion rate by the accumulated mass of clouds per timestep $\Delta t$, which have distances to the Galactic Centre less than 2 pc. This evolution is shown in Fig. 11 for four different energy loss rates per collision: $0 \%, 1 \%, 10 \%$, and an energy loss that is proportional to the fraction of the clouds' mass, which participates in the collision (Krolik \& Begelman 1988). The evolution of the mass accretion rate and the collision rate are similar for all energy loss rates. During the first collision at $t \sim 0.4$ Myr the total accreted mass is $\sim 3000 M_{\odot}$ within 0.1 Myr. Nevertheless, this is not the major accretion event. This happens between 2 and $3 \mathrm{Myr}$, when the tidally stretched cloud (streamer) is crossing itself. The total accreted mass in this second event is $\sim 10^{4} M_{\odot}$ within $2 \mathrm{Myr}$. At later stages of the simulation the mass accretion rate decreases steadily having still some small peaks. The comparison with the collision rate shows that the first accretion event coincides with the the first collision of the cloud with the CND. The major event coincides with the second to fourth collisions and with the first crossing of the streamer with itself. The later and smaller accretion events coincide with later collisions between the streamer and the CND. The peak mass accretion rate at the beginning is $\sim 3 \times 10^{-2} M_{\odot} \mathrm{yr}^{-1}$ falling to several $10^{-4} M_{\odot} \mathrm{yr}^{-1}$ after an elapsed time of $6 \mathrm{Myr}$. The peak of the major accretion event is $\sim 10^{-2} M_{\odot} \mathrm{yr}^{-1}$ decreasing steadily until the end of the simulation.

The evolution of the simulation with $1 \%$ energy loss per collision is almost identical to that without energy loss. With an energy loss rate of $10 \%$ per collision the mass is accreted approximately 2 times faster than for the simulation without energy loss. After the first peak the mass accretion rate stays at a value $>0.01 M_{\odot} \mathrm{yr}^{-1}$. Then it decreases strongly from 2 to $5 \mathrm{Myr}$ and rises again at $9 \mathrm{Myr}$. The collision rate shows the same behaviour. Clouds collide more frequently during 1 and $2 \mathrm{Myr}$ as for the simulation without energy loss. At $t=6 \mathrm{Myr}$ the collision rate begins to rise from $4 \times 10^{-4} \mathrm{yr}^{-1}$ to $1.5 \times 10^{-3} \mathrm{yr}^{-1}$ at $9 \mathrm{Myr}$. This happens, because the cloud distribution of the infalling streamer symmetrizes and forms a second circumnuclear disk with a mass of $\sim 1.5 \times 10^{4} M_{\odot}$.

\subsubsection{Prograde encounter}

The evolution of the system with no loss of kinetic energy per collision can be seen in Fig. 12. The timestep is $\Delta t=1.2 \mathrm{Myr}$. In difference to the retrograde encounter, the CND is not damaged by the infalling cloud. During the evolution of the system the cloud-cloud collisions change the angular momentum of the CND and that of the infalling cloud. With no energy loss per collision this alignment is minimum, i.e. at the end of the simulations the 

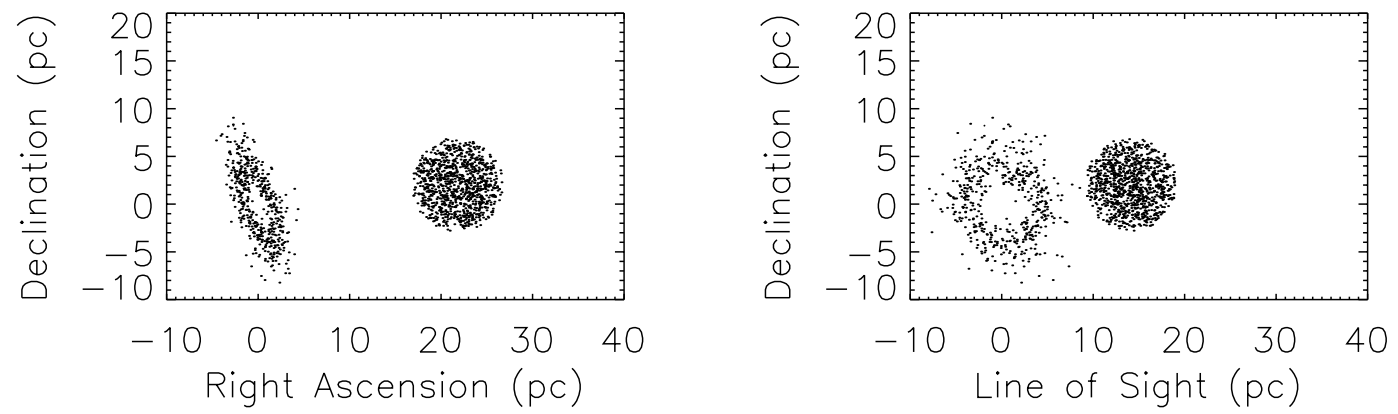

Fig. 9. Initial conditions for the cloud infall into the Galactic Centre. The axis correspond to: left: right ascension, declination; right: LOS (z-axis), declination.

majority of the CND clouds have still their initial angular momentum. We observe at the end of the simulations a triangular structure with an outer ring-like structure and the CND inside. Thus, one can still recognize two structure with different angular momenta. For the simulations with an energy loss greater than $5 \%$ the situation changes. At the end of the simulation there is only one kinematical entity, a new CND with an angular momentum between that of the CND and that of the infalling cloud. The evolution of the system is shown in Fig. 13 for 6 different energy loss rates per collision $\epsilon$ : $0 \%, 1 \%, 5 \%, 10 \%, 20 \%$, and a mass dependent energy loss. Whereas in the case of a retrograde encounter the general behaviour of the different quantities were the same for different energy loss rates, in the case of a prograde encounter these evolutions change dramatically for different energy loss rates. In the case of energy loss rates $\epsilon<1 \%$ the mass accretion rate shows two peaks of $\dot{M} \sim 2 \times 10^{-3} M_{\odot} \mathrm{yr}^{-1}$. After a third small peak the mass accretion rate drops to zero. In the case of $\epsilon=5 \%$ there are also two peaks at the beginning followed by an increase between $t \sim 2 \mathrm{Myr}$ and $4 \mathrm{Myr}$ $\left(\dot{M} \sim 2 \times 10^{-3} M_{\odot} \mathrm{yr}^{-1}\right)$ and decreases then slowly. For $\epsilon=10 \%$ there is a gap in the mass accretion rate after the first collision between the infalling cloud and the CND. At later timesteps the mass accretion rate stays almost constant at a value of $\dot{M} \sim 2 \times 10^{-3} M_{\odot} \mathrm{yr}^{-1}$. The gap disappears for $\epsilon=20 \%$. After a large first maximum between 0.5 and $1.5 \mathrm{Myr}\left(\dot{M} \sim 7 \times 10^{-3} M_{\odot} \mathrm{yr}^{-1}\right)$, the mass accretion rate has a minimum at $t \sim 2.5 \mathrm{Myr}$, rises again to $7 \times 10^{-3} M_{\odot} \mathrm{yr}^{-1}$ at $t \sim 4 \mathrm{Myr}$ and decreases again. In the case of a mass dependent energy loss the mass accretion rate decreases steadily from its initial maximum of $\sim 10^{-2} M_{\odot} \mathrm{yr}^{-1}$ to $\dot{M} \sim 10^{-3} M_{\odot} \mathrm{yr}^{-1}$ after $10 \mathrm{Myr}$.

The evolution of the collision rate also shows big differences for different energy loss rates $\epsilon$. Whereas the peak $\left(t_{\text {coll }}^{-1} \sim 2 \times 10^{-3} \mathrm{yr}^{-1}\right)$ due to the first encounter between the infalling cloud and the CND is always there, the subsequent behaviours are markedly different. For $\epsilon=0 \%$ the collision rate begins to rise at $t=3 \mathrm{Myr}$ until $t=6 \mathrm{Myr}$ and then stays constant $\left(t_{\text {coll }}^{-1} \sim 5 \times 10^{-3} \mathrm{yr}^{-1}\right)$. For $\epsilon=5 \%$ the collision rate begins to rise at $t \sim 1.5 \mathrm{Myr}$ and stays constant between 3 and $10 \mathrm{Myr}\left(t_{\text {coll }}^{-1} \sim 2 \times 10^{-3} \mathrm{yr}^{-1}\right)$. For higher $\epsilon$ the collision rate even decreases at $t \sim 4 \mathrm{Myr}$.

\section{Comparison with the Sgr A cloud complex}

We have constructed a simple toy model in order to compare the observed Sgr A cloud complex (Zylka et al. 1990) to a snapshot of our simulation (for the observational interpretation see Sect. 5). The Sgr A Radio and GMC Complex observed at $\lambda 1.3 \mathrm{~mm}$ with the IRAM MRT (Mezger et al. 1989; Zylka et al. 1990), the synchrotron shell Sgr A East observed at $\lambda 6 \mathrm{~cm}$ with the VLA (Yusef-Zadeh \& Morris 1987), and ${ }^{13} \mathrm{CO}(2-1)$ IRAM spectra (Mezger et al. 1996) are shown in Fig. 14. Since there seems to be at least two different kinematical features, we have added two snapshots of the tidally stretched cloud at two different times (0.6 Myr and 1.6 Myr) together with the CND. This has been done using a simulation with 5 times more clouds in order to have a higher particle resolution. The cloud cross sections were adapted to ensure the same mean collision time scale as for the simulation with 1500 clouds. We use a prograde simulation, because it fits best observed line-of-sight locations of the GMCs in the vicinity of the CND. At these early stages of evolution, the results do only marginally depend on the energy loss rate per collision $\epsilon$. We therefore chose $\epsilon=0$.

This composite snapshot can be seen in the left panel of Fig. 15. It should be stressed here that the aim of this comparison is not to reproduce each feature in detail but to make a generic picture of the Sgr A region. Therefore, it is not troublesome that the cloud in the east of the Galactic Centre is not as close as M-0.02-0.07. However, the CND and the streamer in south-north direction containing M-0.13-0.08 are nicely reproduced. The mass distribution in direction of the line of sight (LOS) is shown in the lower panel of Fig. 15. The streamer in south-north direction is located in front of the Galactic Centre as well as the eastern cloud.

The radial velocity field can be seen in Fig. 16. The velocities in the RA-Dec plane are shown in Fig. 17. The streamer in south-north direction is thus approaching the Galactic Centre from the front crossing the northern part of the CND. The eastern cloud is near the apocenter of its elliptical orbit, the north-western part having already changed the sign of the radial velocity falling onto the CND from the front. 

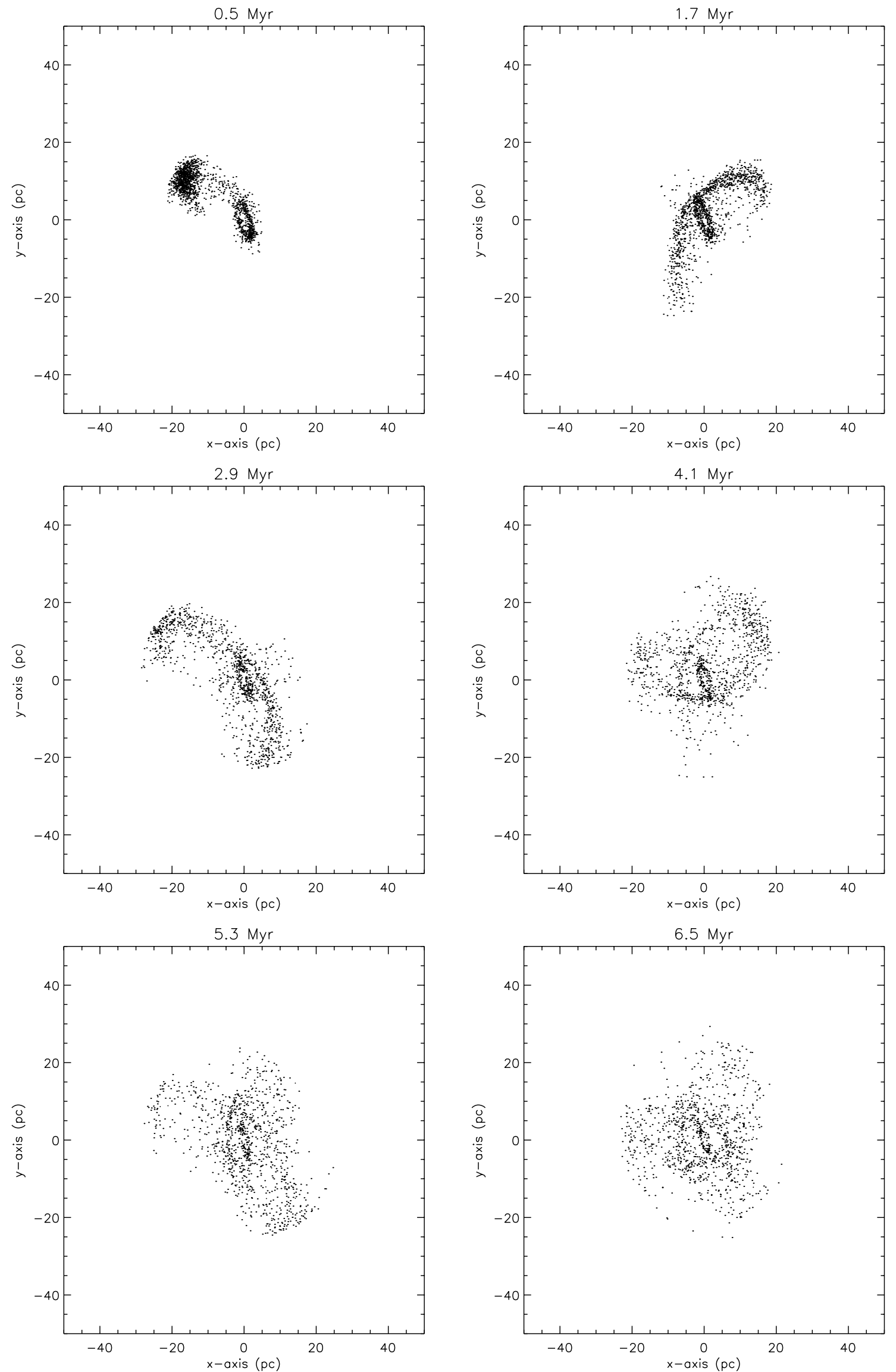

Fig. 10. The evolution of the cloud infall into the Galactic Centre as the observer would see it from the Earth. The elapsed time is plotted on the top of each frame. 

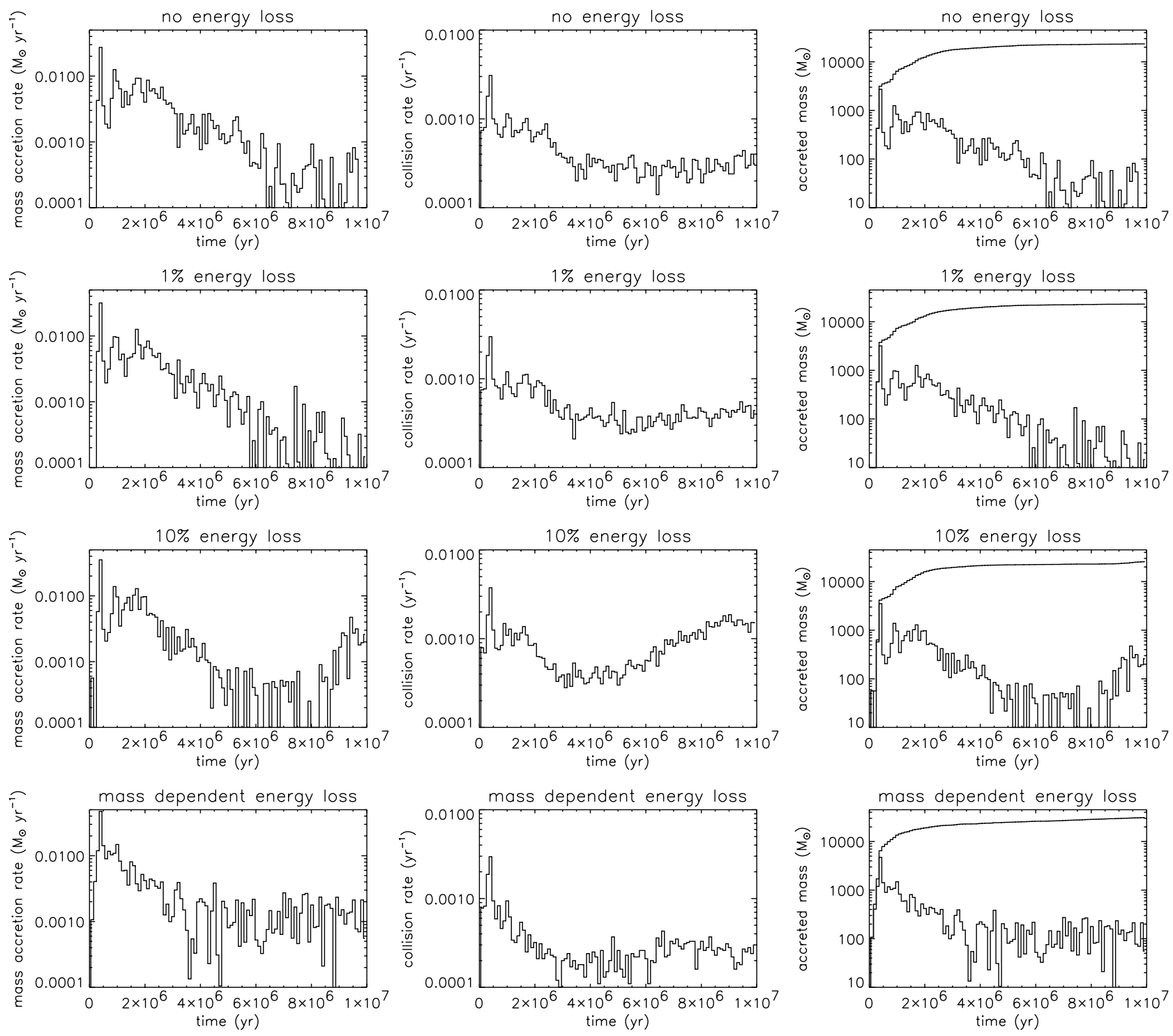

Fig. 11. 1st column: mass accretion rate; 2nd column: number of collisions per timestep $\Delta t$; 3rd column: accreted mass per timestep $\Delta t$ and accumulate accreted mass. Rows: no energy loss, 1\%, 10\%, mass dependent loss of kinetic energy per collision.

Zylka et al. (1998) show CS(3-2) spectral line data observed at the IRAM $30 \mathrm{~m}$ Telescope covering the central $21^{\prime} \times 20^{\prime}$ with a spatial resolution of $17^{\prime \prime}$. Their galactic longitude-velocity and galactic latitude-velocity plots can be directly compared with our simulations. We therefore made plots of exactly the same slices in $\Delta l$ and $\Delta b$ respectively.

The galactic longitude-velocity plots of Zylka et al. (1998) are plotted in Fig. 18. The corresponding model diagrams can be seen in Fig. 19. The main features of the $\operatorname{CS}(3-2)(v-\Delta l)$ plots are reproduced by the model. Obviously, the CS(3-2) intensity does not match the column density of the model plots. This might be due to the fact that the $\mathrm{CS}(3-2)$ emission depends on the physical condition out of which only one is the total column density. In Fig. 19a the streamer in south-north direction is clearly visible. Nevertheless it appears more prominent in the corresponding CS(3-2) plot (Fig. 18a). In Figs. 19b and $\mathrm{c}$ the CND is prominent. We miss the observed prominent cloud complex at positive velocities and positive longitudes almost completely. The eastern model streamer can be seen in in the upper right part of Fig. 19a and as an extension of the CND structure at positive velocities in Fig. 19b. Both features have a counterpart in Figs. 18a and $\mathrm{b}$. In contrast, the feature at negative velocities in Fig. 19d, which is due to the eastern part of the eastern streamer (Fig. 16), in Fig. 19d has no counterpart in Fig. 18d. This implies that the observations might correspond to a slightly later timestep in the evolution of the eastern model cloud and/or that its orbital parameters might be slightly different from that of the observed cloud. Nevertheless, our eastern model cloud represents a valuable first approach in modelling M-0.02-0.07. 

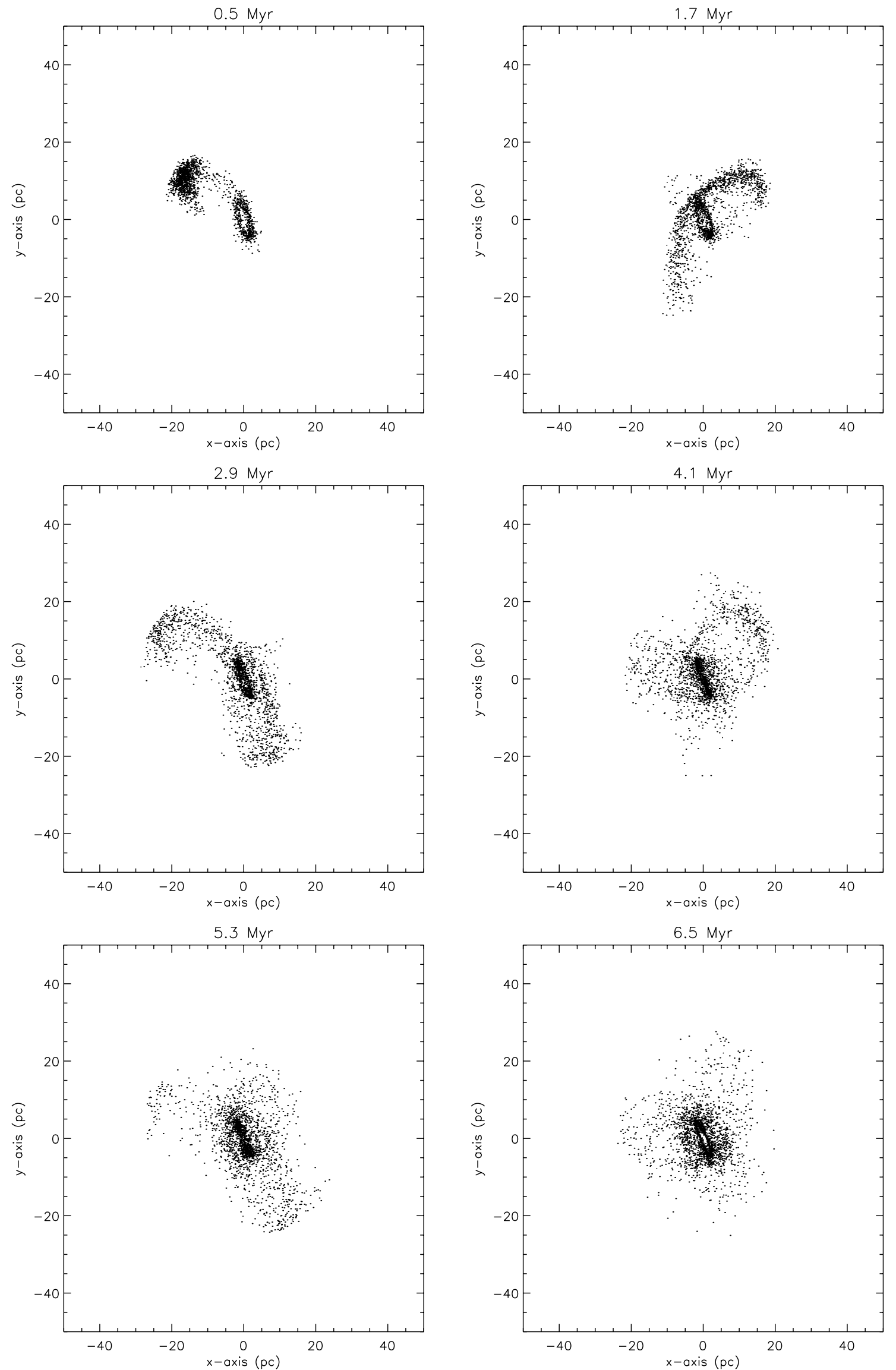

Fig. 12. The evolution of the cloud infall into the Galactic Centre as the observer would see it from the Earth. The elapsed time is plotted on the top of each frame. 

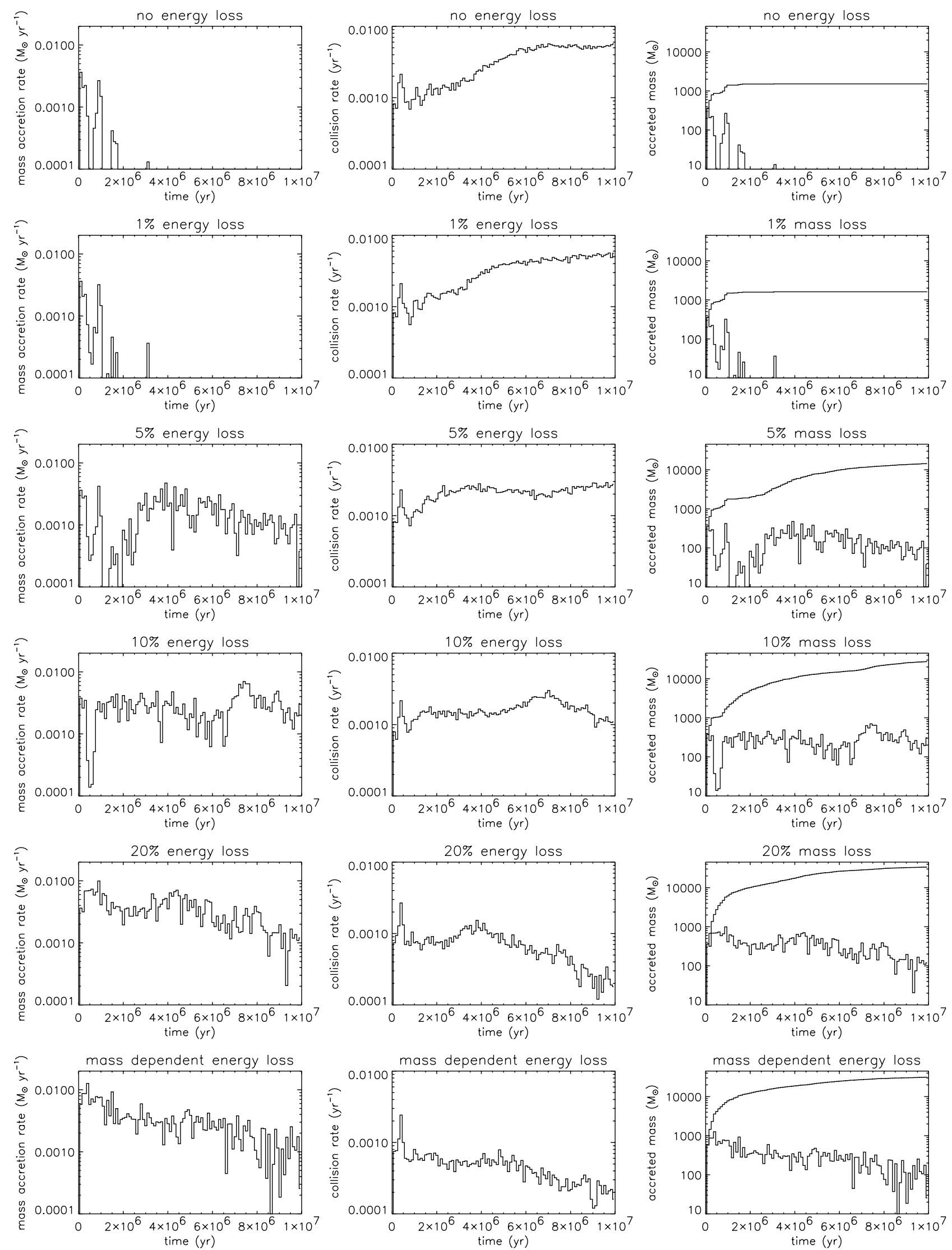

Fig. 13. 1st column: mass accretion rate; 2nd column: number of collisions per timestep $\Delta t$; 3rd column: accreted mass per timestep $\Delta t$ and accumulate accreted mass. Rows: no energy loss, 1\%, $5 \%, 10 \%, 20 \%$, mass dependent loss of kinetic energy per collision. 


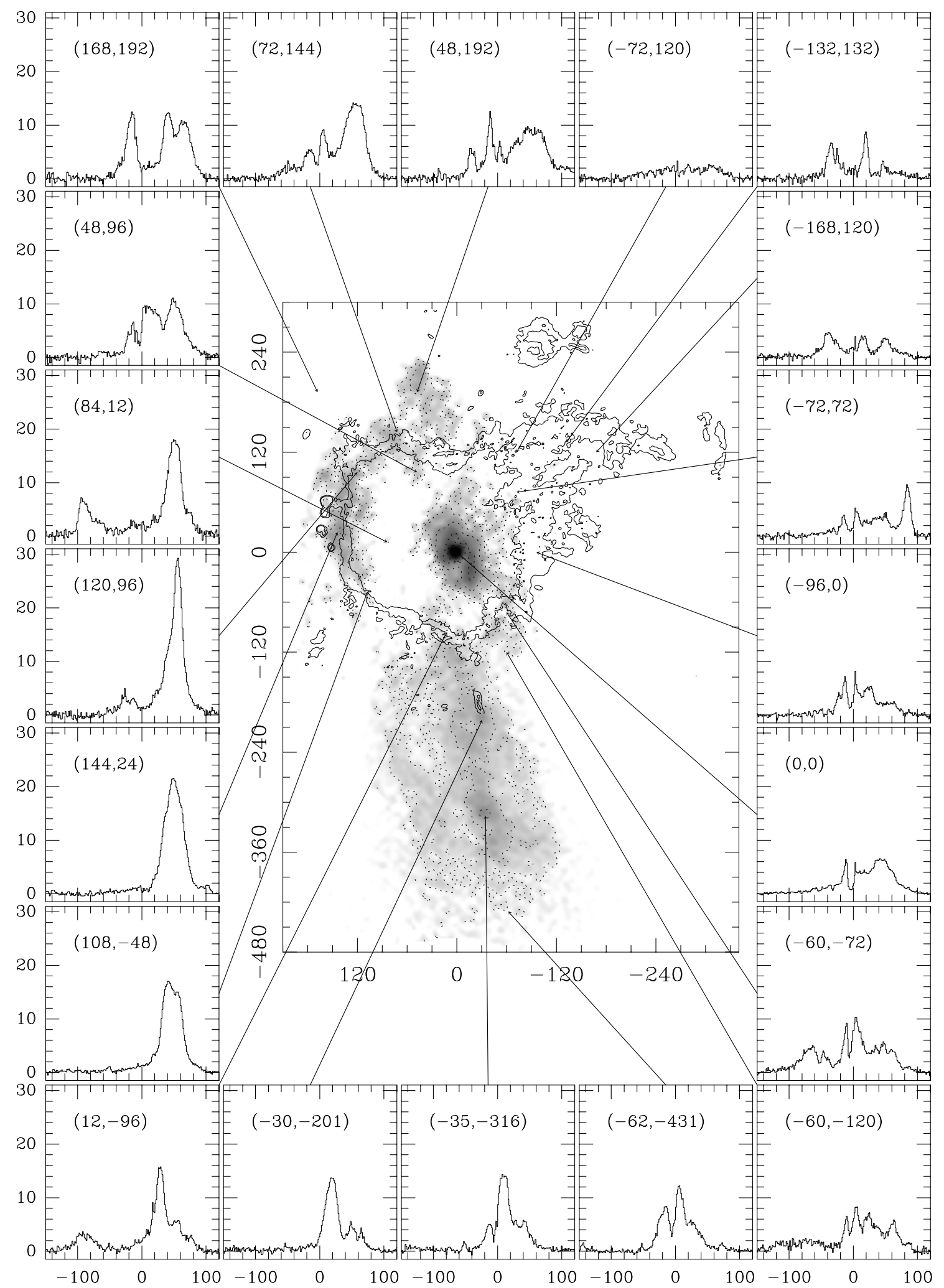

Fig. 14. The Sgr A Radio and GMC Complex. Greyscale: $\lambda 1.3 \mathrm{~mm}$ with the IRAM MRT (Mezger et al. 1989; Zylka et al. 1990). Contour: the synchrotron shell Sgr A East observed at $\lambda 6 \mathrm{~cm}$ with the VLA (Yusef-Zadeh \& Morris 1987). Frame panels: ${ }^{13} \mathrm{CO}(2-1)$ IRAM spectra (Mezger et al. 1996).

The galactic latitude-velocity plots of Zylka et al. (1998) are plotted in Fig. 20. The corresponding model diagrams can be seen in Fig. 21. We succeed in reproducing the feature at negative latitudes and positive velocities (Figs. 21e and $\mathrm{f}$ ), which is caused by the southern streamer. The model velocities are offset by 

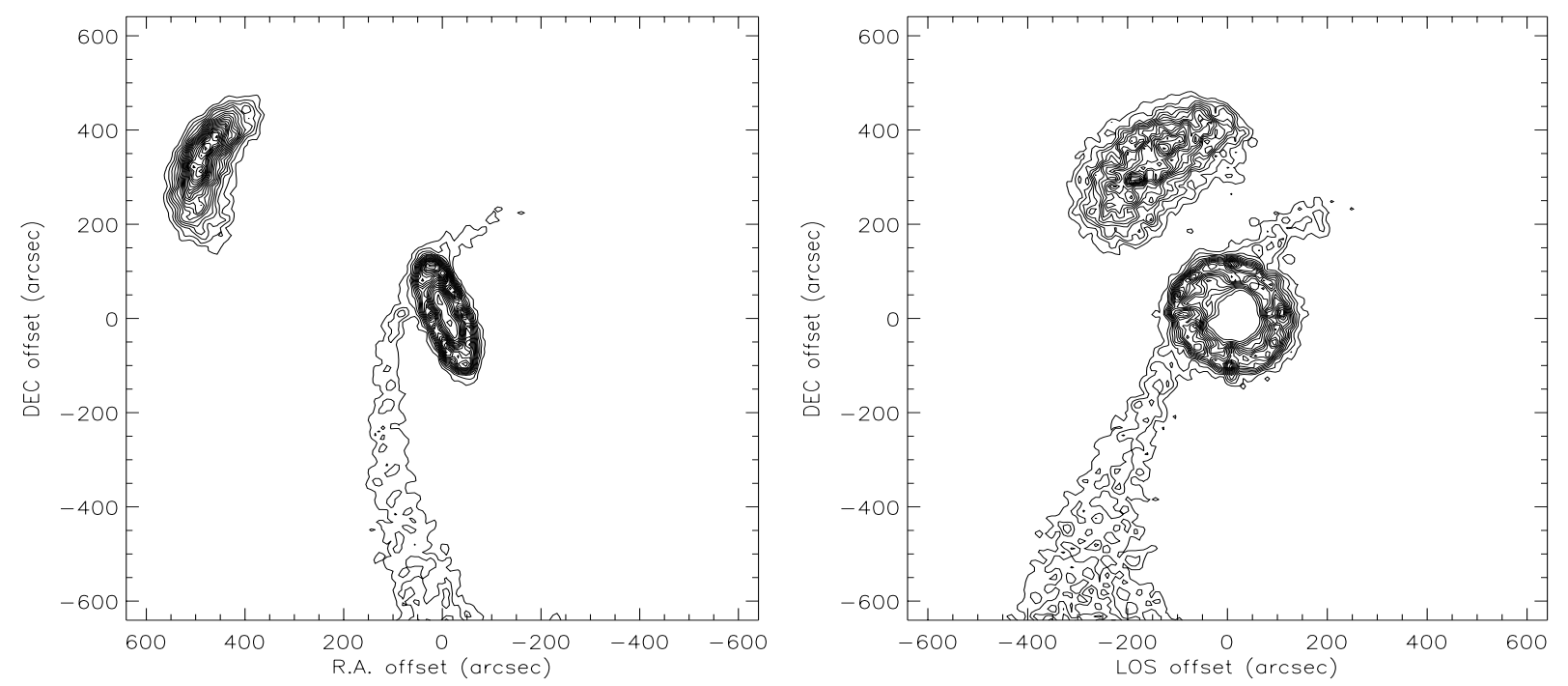

Fig. 15. Left panel: combined snapshots of the high mass simulation for the comparison with the Sgr A cloud complex. Right panel: the same mass distribution but with the line of sight (LOS) as the $y$-axis. Smaller distances are nearer to the observer.

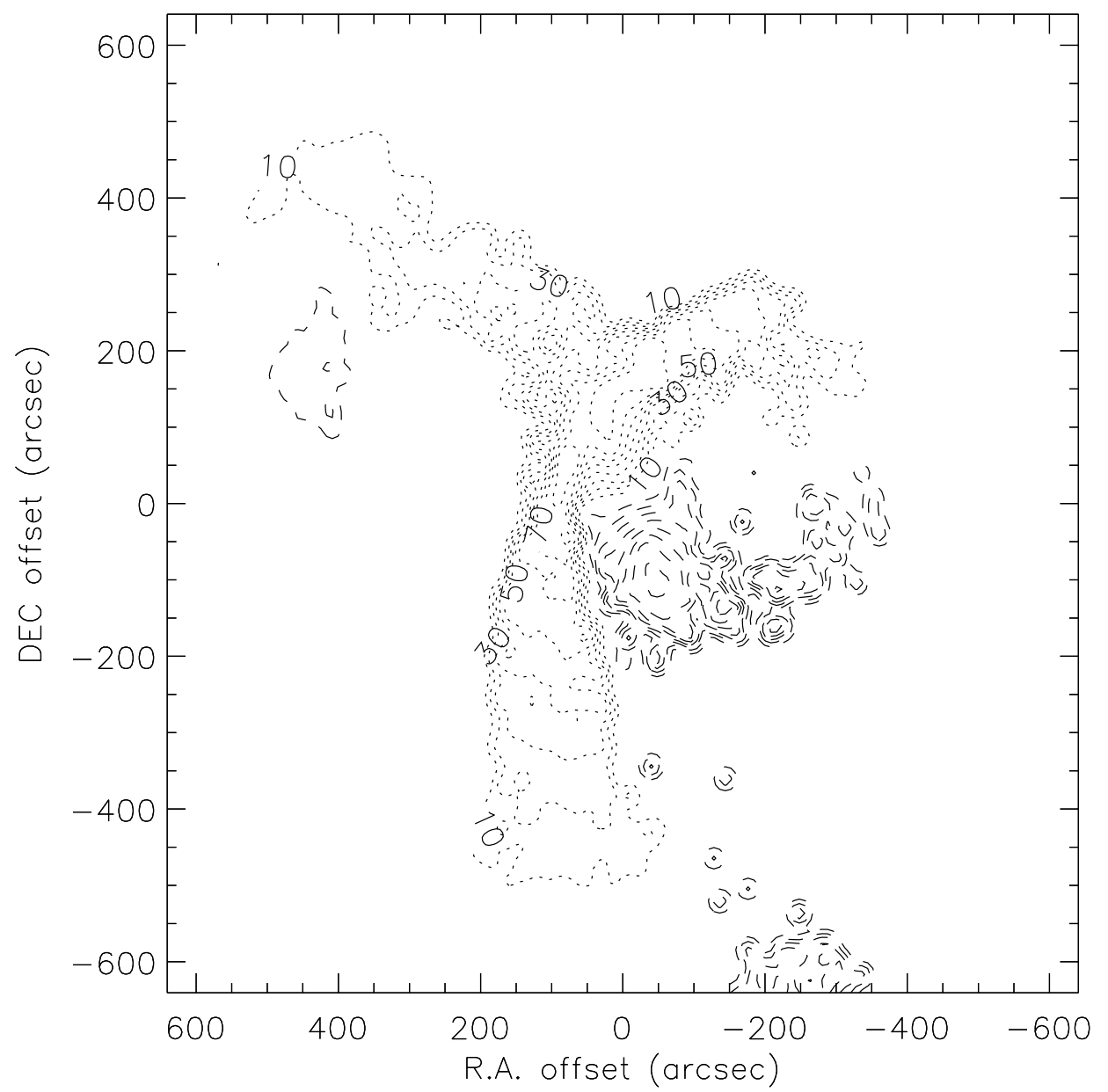

Fig. 16. The radial velocity field of Fig. 15. Dashed lines represent negative velocities, dotted lines positive velocities. The contours are in the range of $-120-120 \mathrm{~km} \mathrm{~s}^{-1}$ with a stepsize of $10 \mathrm{~km} \mathrm{~s}^{-1}$.

$\sim 40 \mathrm{~km} \mathrm{~s}^{-1}$ from the observed ones in both diagrams. The eastern streamer, which can be seen at negative latitudes in Figs. 21a and b has only an observed counterpart in
Fig. 20b. This is due to the fact that the eastern model streamer is located more to the north than M-0.02-0.07. The additional CND features at negative latitudes and 


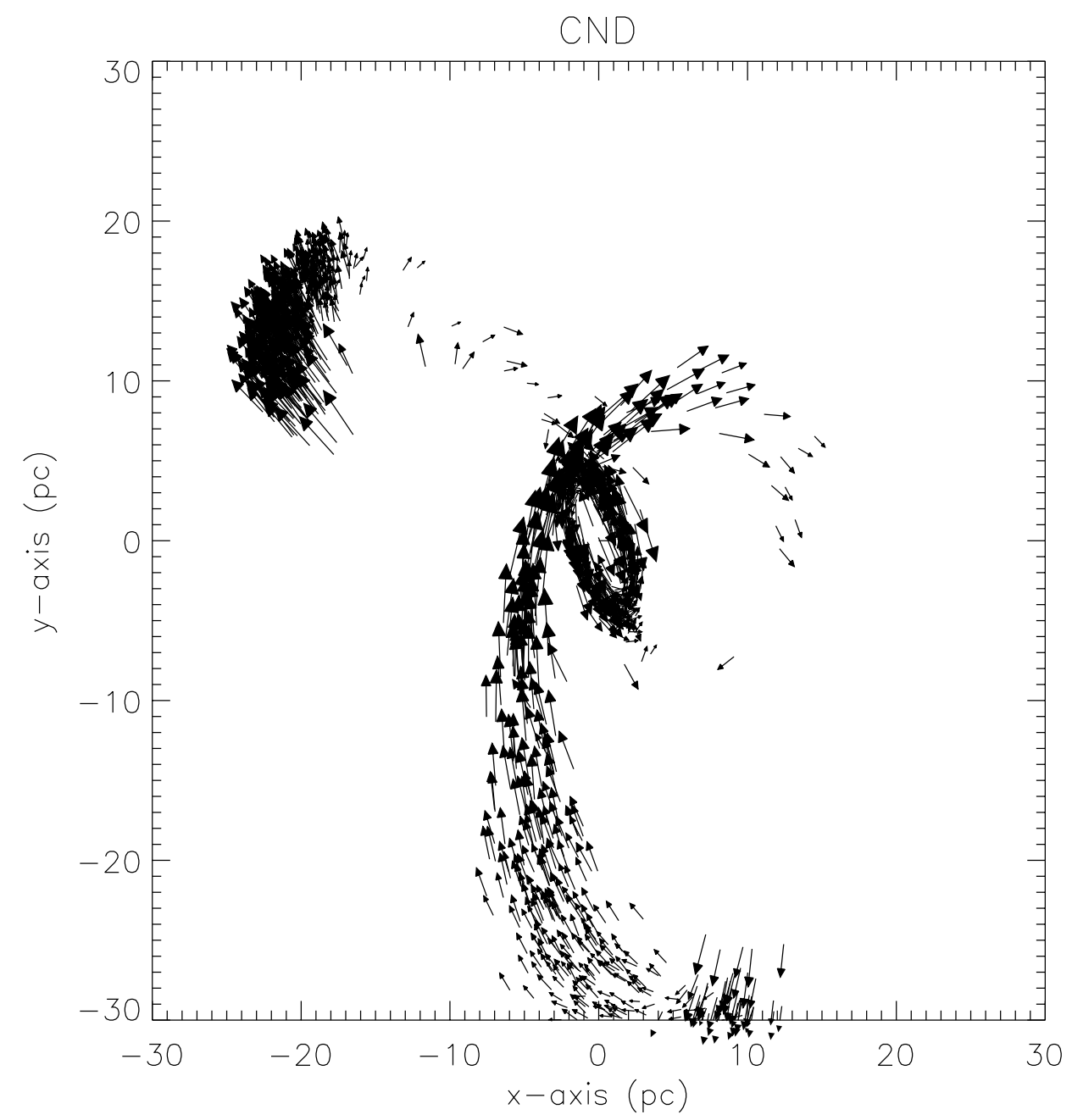

Fig. 17. The velocity field of Fig. 15 in the RA-Dec plane. For clarity only each 10th velocity vector is plotted. The CND is rotating counter clockwise. Its outer rim is rotating clockwise.

negative velocities (Figs. 21c and d) might indicate that the position angle of the CND might differ by some degrees between the model and the real CND.

\section{Discussion}

In this work we investigated the dynamical behaviour of the CND in the Galactic Centre. In a first step we treated an isolated ring-like structure which contains 500 clouds with a cloud mass of $\sim 30 M_{\odot}$. Once in equilibrium, the disk shows the same height with respect to the radius as it is deduced from mm-observations (Güsten et al. 1987). We normalized the number of collisions per unit time with the help of a simple spherical cloud distribution. The resulting value for the CND simulation in equilibrium is $t_{\text {coll }} \sim 2 \times 10^{6}$ yr. Therefore, the isolated CND can be longer lived as it was thought before. This collision time scale implies a mass accretion rate of several $10^{-4} M_{\odot} \mathrm{yr}^{-1}$. We conclude that the current mass accretion rate at pc scale ranges between $10^{-3}$ and $10^{-4} M_{\odot} \mathrm{yr}^{-1}$ (the latter being the value for the isolated CND, see Vollmer \& Duschl 2001a).
In a second step the environment of the CND was taken into account. We have constructed a dynamical model where a spherical cloud falls into the Galactic Centre onto a pre-existing CND. A retrograde and a prograde encounter were simulated with different kinetic energy loss rates $\epsilon$ per collisions. In the case of a retrograde encounter the evolution of the mass accretion and cloud collision rate during the collision between the cloud and the CND are not very sensitive to $\epsilon$. We observe a first maximum during $\Delta t \sim 0.1$ Myr followed by a larger maximum $(\Delta t>1 \mathrm{Myr})$. In the case of a prograde encounter we observe dramatic changes in the behaviour of these quantities. For small energy loss rates per collision $(\epsilon \leq 2 \%)$ only $\sim 2 \times 10^{3} M_{\odot}$ are accreted within less than $2 \mathrm{Myr}$, whereas the collision rate increases by a factor of $\sim 5$ between 2 and 5 Myr. For intermediate energy loss rates $(5 \% \leq \epsilon \leq 10 \%)$ we observe an approximately constant mass accretion rate of $\dot{M} \sim 3 \times 10^{-3} M_{\odot} \mathrm{yr}^{-1}$ with a gap $(\Delta t \sim 0.5 \mathrm{Myr})$ after the first encounter of the cloud with the CND. For $\epsilon=5 \%$ there is second broader gap $(\Delta t \sim 2 \mathrm{Myr}) \sim 1 \mathrm{Myr}$ after the first collision of the cloud with the CND. The collision rate between the clouds stays 


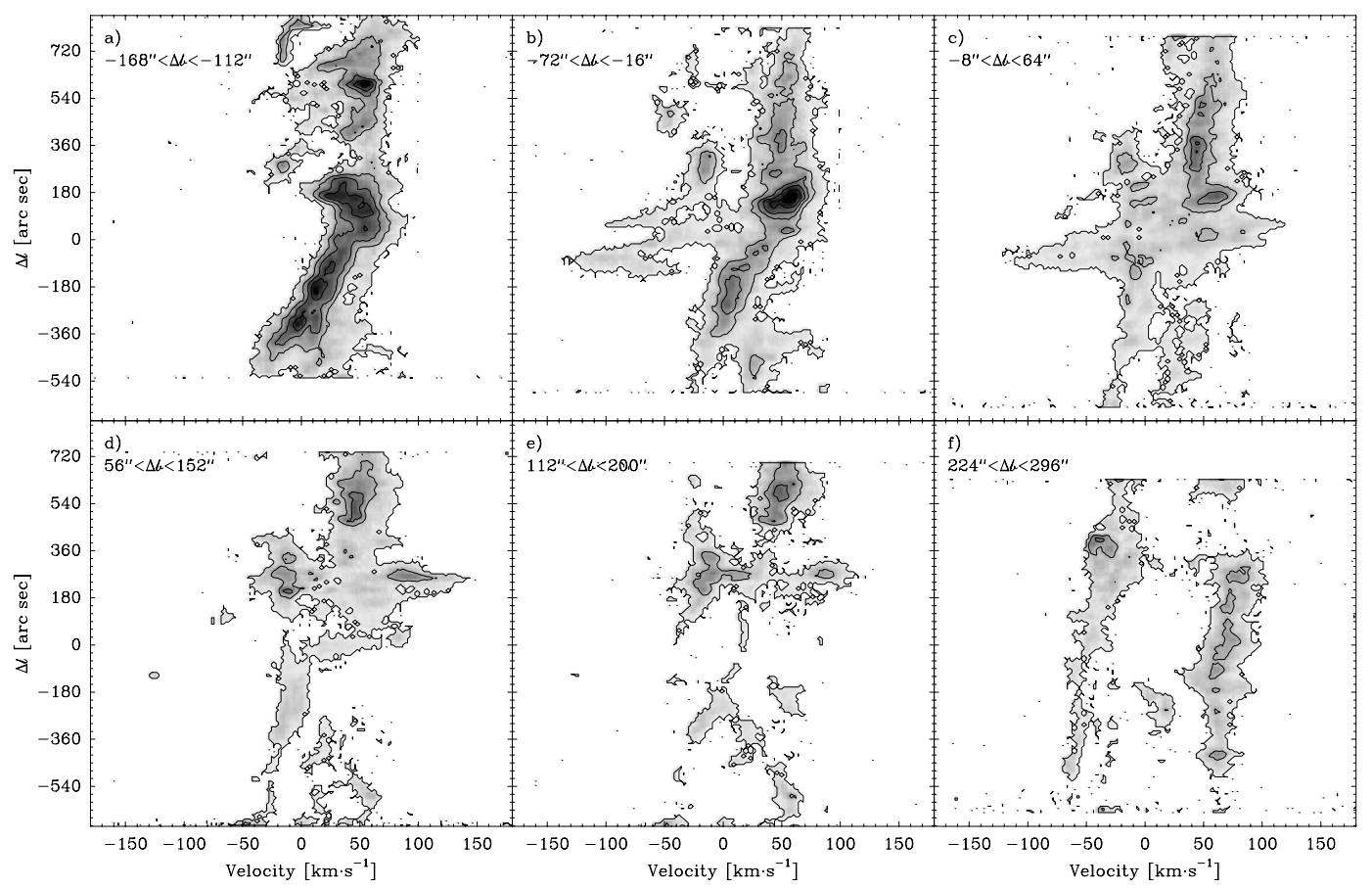

Fig. 18. Representative galactic longitude-velocity plots $(v-\Delta l)$ of the $\operatorname{CS}(3-2)$ emission. The data have been averaged over selected ranges in galactic latitudes, $\Delta b$ (Zylka et al. 1998).
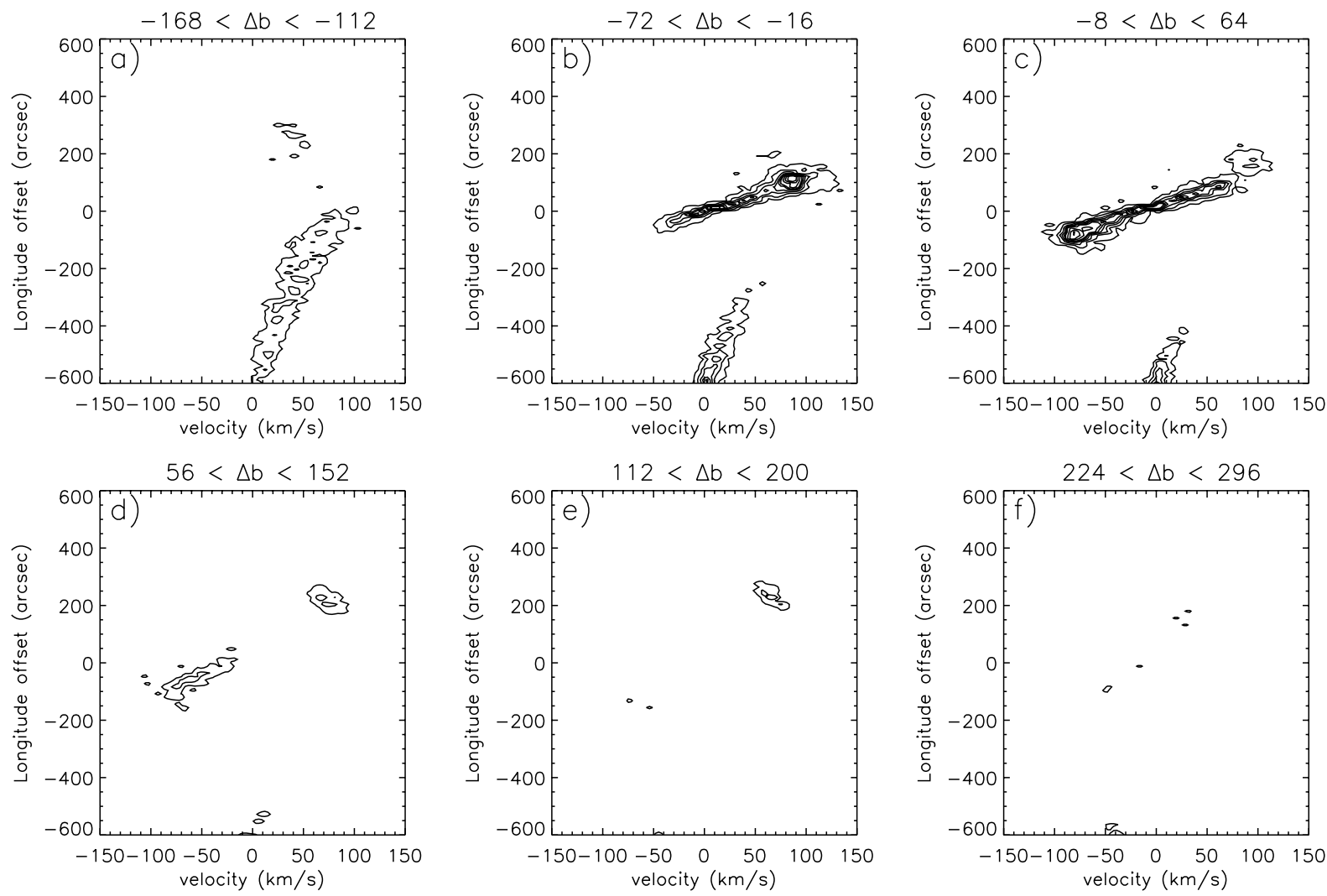

Fig. 19. Corresponding galactic longitude-velocity model plots $(v-\Delta l)$.

constant $\left(t_{\text {coll }}^{-1} \sim 3 \times 10^{-3} \mathrm{yr}^{-1}\right)$ over the whole evolution. For $\epsilon \geq 20 \%$ the initial value of the mass accretion rate is more than a factor of 2 higher than for $\epsilon<$ $20 \%$ and decreases steadily during the whole evolution.
The collision rate shows a prominent peak when the cloud collides for the first time with the CND, drops then to $t_{\text {coll }}^{-1} \sim 10^{-3} \mathrm{yr}^{-1}$ and varies by a small amount during the further evolution. 


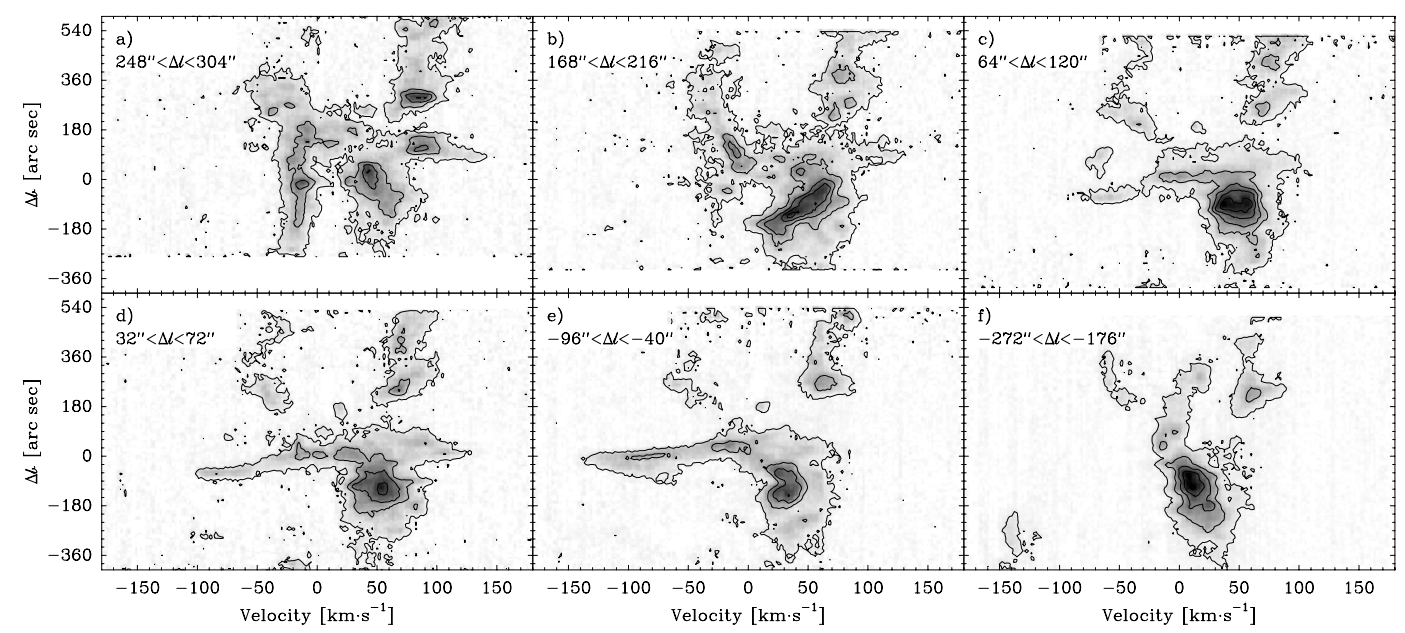

Fig. 20. Representative galactic latitude-velocity plots $(v-\Delta b)$ of the $\operatorname{CS}(3-2)$ emission. The data have been averaged over selected ranges in galactic longitude, $\Delta l$ (Zylka et al. 1998).
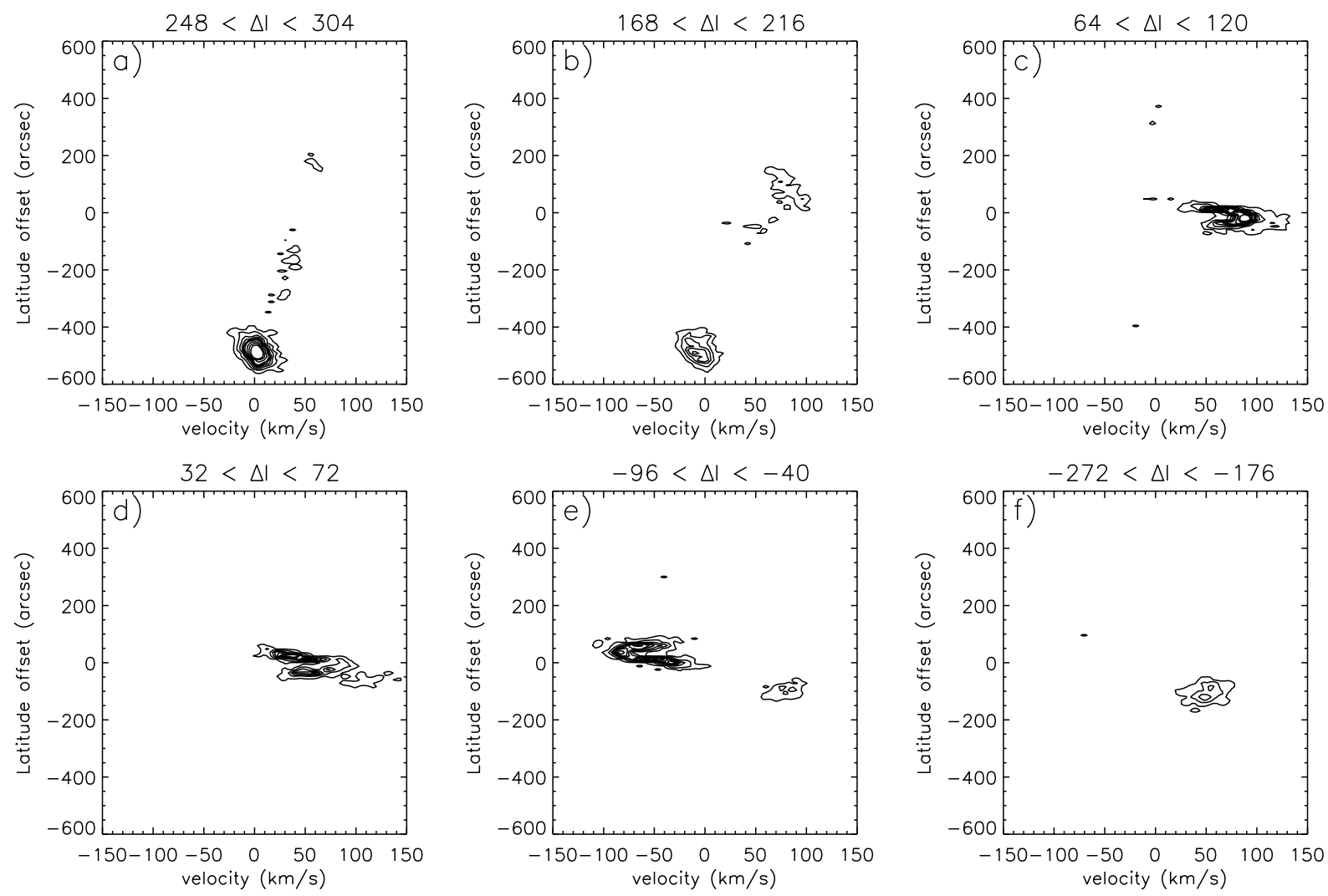

Fig. 21. Corresponding galactic latitude-velocity model plots $(v-\Delta b)$.

This strong dependence of the mass accretion and the collision rate on the energy loss rate per collision shows the importance of cooling mecanisms and the magnetic field, which both influence the energy loss rate. Whereas the OI and CII lines are the major contributors to radiative energy losses in the shock front during the collision, the magnetic field retains kinetic energy in the clouds. Following Krolik \& Begelman (1988) the loss rate of kinetic energy during a cloud-cloud collision is given by $\epsilon=f \xi$, where $f$ is the fraction of cloud's mass participating in the collision and $\xi$ is the degree of inelasticity.
Krolik \& Begelman (1988) estimate $f=0.2$, which is in good agreement with our findings for the simulations with a mass dependent energy loss rate. The degree of inelasticity is given by

$\xi=1-\frac{1}{\mathcal{M} \sqrt{\beta}-\frac{1}{2}}$,

where $\mathcal{M}$ is the Mach number and $\beta$ is the ratio between gas and magnetic pressure. A Mach number $\mathcal{M} \sim 20$ and $\beta \sim 1$ gives $\xi=0.95$. Only for $\beta<0.1$ more than $20 \%$ of the energy available in the collision is retained by the magnetic field. The strength of the magnetic field in the CND 
is about $1 \mathrm{mG}$ (Yusef-Zadeh et al. 1996), the cloud densities are of the order $10^{6} \mathrm{~cm}^{-3}$, and the gas temperature within the clouds is $\sim 150 \mathrm{~K}$ (Vollmer \& Duschl 2001a). This leads to $\beta$ of the order of 1 . Therefore, we conclude that magnetic fields might not play a preponderant rôle for the evolution of the CND as long as Eq. (7) holds.

Vollmer \& Duschl (2001a) estimate the energy loss rate in the shock during the collisions to be $\epsilon=10 \%$. Thus, most realistic simulations are those with $\epsilon=10-20 \%$ and a mass dependent energy loss rate (lower panels in Figs. 11 and 13). For $\epsilon>10 \%$ the evolutions of the mass accretion and cloud collision rates of a retrograde and a prograde encounter are very similar: there is a prominent peak after the first collision between the cloud and the CND followed by steady decrease. For $\epsilon=10 \%$ there are measurable differences between a retrograde and a prograde encounter (see Sect. 5.2).

In a further step we have constructed the observed features of the Sgr A cloud complex by adding two snapshots of our simulations. We can reproduce the observed topography in a satisfying way in the RA-Dec plane and in galactic longitude/latitude-velocity planes. This means that the orbit of the recently infalling model cloud is prograde with respect to the CND and the gas motion in the galactic disk. The observed warp of the CND (Güsten et al. 1987) can be naturally explained by a former prograde encounter (see Sect. 5.2.2). Based on our model, there is the possibility that one of the streamers has already passed the Galactic Centre less than 0.5 Myr years ago. During this former interaction of a cloud and the CND a small starburst might have taken place at a distance of several pc from the Galactic Centre. Since the front end of the tidally stretched cloud and the front of the eastern streamer are just crossing the CND today, we should be able to find signs of a small current starburst in the CND. This might be the case for the observed Or cloud (the Tongue, Jackson et al. 1993). In this case, the incoming streamer hits the CND at $\sim 3-5$ pc. The clouds lose angular momentum due to collisions and are contracted. The so infalling perturbed clouds fragment, collapse, and form stars. These new ionizing $\mathrm{O}$ and $\mathrm{B}$ stars are still embedded in the part of the cloud from which they formed. Ott et al. (1999) found evidence for ongoing star formation in the Central Cavity. Especially, they identified stars embedded in extended dust clouds within the Minispiral. The UV radiation of the young hot stellar population can penetrate efficiently into the fragmented cloud. Therefore, the gas in this region is predominantly in atomic form and can not be observed in HCN.

The remaining question is: how and when did the HeI star cluster form? Genzel et al. (1996) found that the HeI stars are rotating in a direction opposite to that of Galactic rotation. If the star cluster was formed through an CND - molecular cloud interaction and if the stars' angular momentum reflects that of their parent cloud, this would imply that the colliding cloud was on a retrograde orbit. Since we have argued that the energy mass loss rate $\epsilon \geq 10 \%$ the two last two rows of Fig. 11 are relevant.
If we assume that star formation is triggered by cloudcloud collisions, the majority of stars is formed during the first $2 \mathrm{Myr}$ after the first collision between the cloud and the CND. Assuming a cloud mass of $30 M_{\odot}$ this leads to a total mass of colliding clouds of $\sim 6 \times 10^{4} M_{\odot}$ within 2 Myr. On the other hand, if the starburst produces stars with a given IMF, we can calculate the initial total gas mass needed to form the observed HeI stars. Assuming an initial mass function $\Phi=C M^{-2}$ with cut-offs at $0.2 M_{\odot}$ and $50 M_{\odot}$ and normalized by the observed HeII stars:

$M_{\mathrm{tot}}^{\mathrm{HeII}}=\int_{10 M_{\odot}}^{50 M_{\odot}} \Phi(M) \mathrm{d} M=25 \times M^{\mathrm{HeII}} \simeq 500 M_{\odot}$

we deduce total stellar mass produced by the starburst of several $10^{4} M_{\odot}$. This would imply that $30-50 \%$ of the colliding gas mass is transformed into stars. Since the cloud orbit is retrograde with respect to the rotation of the CND, the colliding clouds lose efficiently angular momentum and thus fall into the Galactic Centre. Therefore, we expect that the stars are formed near the inner edge of the CND. They need $\sim 7$ Myr to build a centrally peaked spatial distribution (Vollmer \& Duschl 2001b). After this time the initial cloud has the appearance of several streamers (see Fig. 10 at $t=7 \mathrm{Myr}$ ) and can not be recognized any more as one single cloud. Nevertheless, we can imagine that a part of Sgr A East Core could be identified as this remaining feature. In order to give an example for the dynamical behaviour of the system after $10 \mathrm{Myr}$ we plot the velocity field of our simulation after this time (Fig. 22). The mass distribution and the velocity field are complicated with one side approaching the observing and the other one receding. The position and inclination angle of the orbit of the infalling cloud might not be the one observed. Since it is difficult to separate really distinct kinematical features in the observations, the discussed model offers only one plausible explanation for the complicated kinematical structure of Sgr A East Core.

Our model still contains great simplifications concerning the physics of the cloud-cloud collisions (see Sect. 2). The effects of these collisions on the cloud population might change, for example, with a better knowledge of the detailed radiative magneto-hydrodynamics which is clearly needed.

The shortcomings concerning the large scale dynamics of our model can in principle be overcome by modifying the initial conditions for the infalling cloud. But, since the parameter space for these initial conditions is very large, it is not the aim of this work to reproduce each feature in great detail but to show general effects. Nevertheless, we can conclude that the Sgr A cloud complex consists of more than one dynamically independent feature.

For the distinction of the dynamically different features in the Sgr A cloud complex we followed Zylka et al. (1990) who made this distinction on observational grounds. It is still possible that interferences of these features exist. Only an analysis of a complete data cube of this region together with the model calculation can shed more light on this question. 


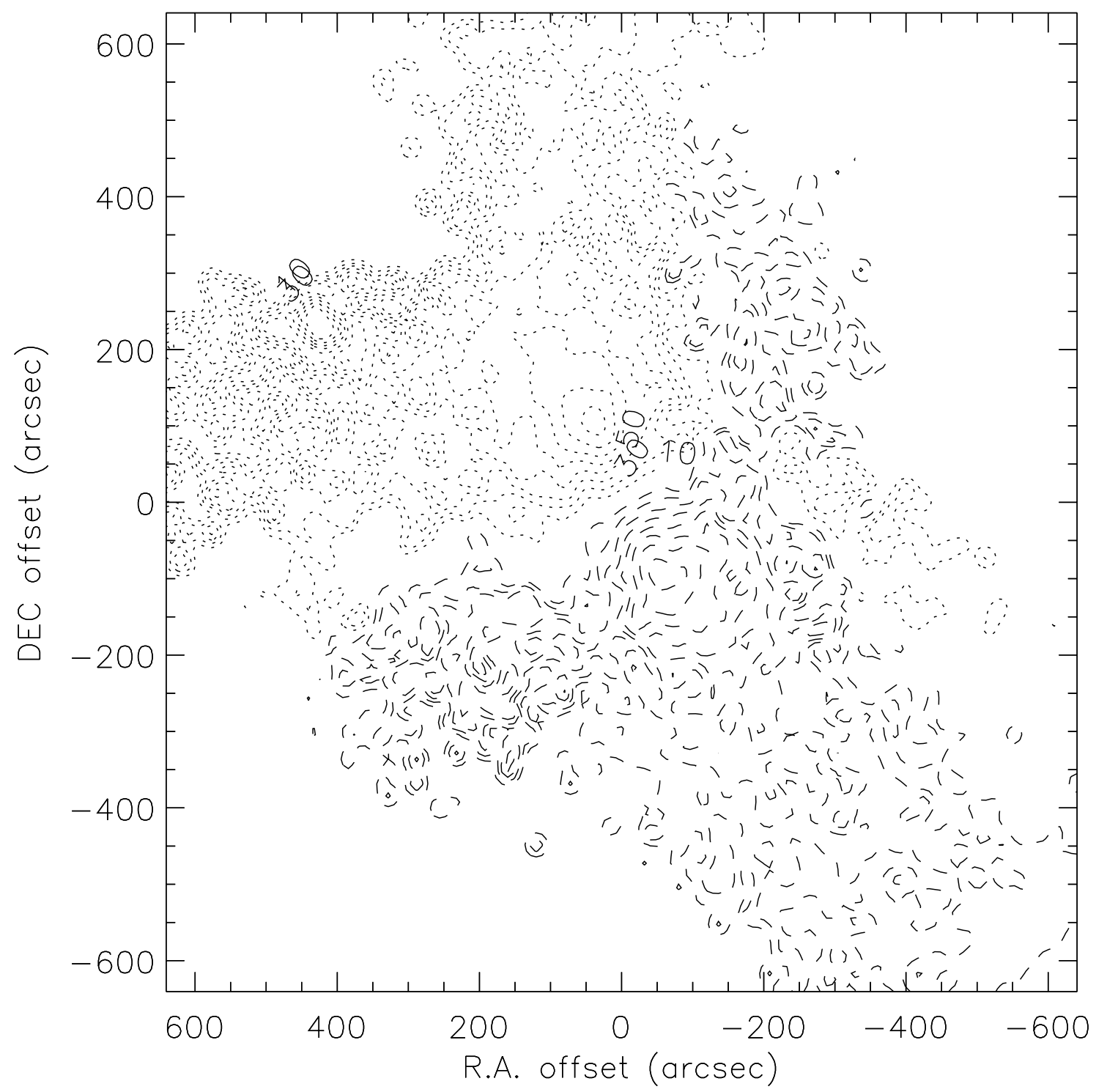

Fig. 22. Velocity field after 10 Myr. This configuration can be compared to the velocity field of the molecular cloud Sgr A East Core. The dotted lines represent positive radial velocities. The dashed lines represent negative radial velocities.

\section{Conclusions}

We have made numerical simulations using a collisional $N$-body code where each particle has a given mass and radius. These clouds can have inelastic that are described schematically. We have first normalized the collision time scale in our code using a spherical configuration. Simulations of an isolated disk structure corresponding to the Circumnuclear Disk (CND) in the Galactic Centre yields a mean collision time scale of one cloud of $t_{\text {coll }} \sim$ 2 Myr and a mass accretion rate of $10^{-4} M_{\odot} \mathrm{yr}^{-1} \leq \dot{M} \leq$ $10^{-3} M_{\odot} \mathrm{yr}^{-1}$.

Since two giant molecular clouds (GMC) are located closer than $50 \mathrm{pc}$ from the CND, we take in a second step this environment of the CND into account. We made simulations for a retrograde and a prograde encounter using different loss rates of kinetic energy during the collisions.
We found that the influence of the energy loss rate on the mass accretion and cloud collision rates is strongest for a prograde encounter. We estimate the energy loss rate per collision of the clouds in the Galactic Centre to be of the order of $10-20 \%$ of the kinetic energy.

The direct comparison of a $1.2 \mathrm{~mm}$ maps and CS(32) position-velocity diagrams of a region containing the CND and the GMCs with a model snapshot shows that the GMCs are on a prograde orbit with respect to the rotation of the CND. A former prograde encounter of a infalling cloud with the CND can naturally explain its observed warp. Since both GMCs might begin to collide with the CND we expect small current star formation near the inner edge of the CND.

Within our scenario of an encounter between an infalling molecular cloud and a CND, we point out the possibility that the HeII star cluster has been formed by a 
retrograde encounter of a cloud with the $\mathrm{CND} \sim 7 \mathrm{Myr}$ ago. The cloud that formed the HeII star cluster has been destroyed by tidal forces and can presently no longer be distinguished as one single kinematical entity.

\section{References}

Barnes, J. E., \& Hut, P. 1986, Nature, 324, 446

Coil, A. L., \& Ho, T. P. T. 1999, ApJ, 513, 752

Coil, A. L., \& Ho, T. P. T. 2000, ApJ, 533, 245

Dent, W. R. F., Matthews, H. E., Wade, R., \& Duncan, W. D. 1993, ApJ, 410, 650

DePoy, D. L., Gatley, I., \& McLean, I. S. 1989, IAU Symp., 136,361

Eckart, A., \& Genzel, R. 1996, Nature, 383, 415

Ekers, R. D., Goss, W. M., Schwarz, U. J., Downes, D., \& Rogstad, D. H. 1975, A\&A, 43, 159

Gatley, I., Jones, J. J., Hyland, A. R., et al. 1986, MNRAS, 222,299

Genzel, R., Thatte, N., Krabbe, A., et al. 1996, ApJ, 472, 153

Güsten, R., Genzel, R., Wright, M. C. H., et al. 1987, ApJ, 318,124

Jackson, J. M., Geis, N., Genzel, R., et al. 1993, ApJ, 402, 173

Krolik, J. H., \& Begelman, M. C. 1988, ApJ, 329, 702

Lacy, J. H., Achtermann, J. M., \& Serabyn, E. 1991, ApJ, 380, L71

Lo, K. Y., \& Claussen, M. J. 1983, Nature, 306, 647

Marr, J. M., Wright, M. C. H., \& Backer, D. C. 1993, ApJ, 411,667
Mezger, P. G., Zylka, R., Salter, C. J., et al. 1989, A\&A, 209, 337

Mezger, P. G., Duschl, W. J., \& Zylka, R. 1996, ARA\&A, 7, 289

Ott, T., Eckart, A., \& Genzel, R. 1999, A\&A, 523, 248

Pringle, J. E. 1981, ARA\&A, 19, 137

Sanders, R. H. 1998, MNRAS, 294, 35

Serabyn, E., Güsten, R., Walmsley, C. M., et al. 1986, A\&A, 169,85

Shlosman, I., Begelman, M. C., \& Frank, J. 1990, Nature, 345, 679

Stoer, J., \& Burlisch, R. 1980, Introduction to Numerical Analysis (New York: Springer-Verlag), chapter 7

Sutton, E. C., Danchi, W. C., Jaminet, P. A., \& Masson, C. R. 1990, ApJ, 348, 503

Vollmer, B., \& Duschl, W. J. 2001a, A\&A, 367, 72

Vollmer, B., \& Duschl, W. J. 2001b, A\&A, 377, 1016

Vollmer, B., \& Beckert, T. 2002, A\&A, 382, 872

von Linden, S., Duschl, W. J., \& Biermann, P. L. 1993, A\&A, 269, 169

Wiegel, W. 1994, Diploma Thesis, University of Heidelberg

Wright, M. C. H., Coil, A. L., McGary, R. S., Ho, P. T. P., \& Harris, A. I. 2001, ApJ, 551, 254

Yusef-Zadeh, F., \& Morris, M. 1987, ApJ, 320, 545

Yusef-Zadeh, F., Roberts, D. A., Goss, W. M., Frail, D. A., \& Green, A. J. 1996, ApJ, 466, L25

Zylka, R., Mezger, P. G., \& Wink, J. E. 1990, A\&A, 234, 133

Zylka, R., Güsten, R., Philipp, S., et al. 1998, in The Central Parsecs, Galactic Center Workshop 1998, ed. H. Falcke, A. Cotera, W. Duschl, F. Melia, \& M. Rieke, 415 